\title{
Judgment Aggregation Rules Based on Minimization
}

\section{Extended Version*}

\author{
Jérôme Lang \\ LAMSADE, Université Paris-Dauphine \\ lang@lamsade.dauphine.fr \\ Gabriella Pigozzi \\ LAMSADE, Université Paris-Dauphine \\ gabriella.pigozzidauphine.fr \\ Marija Slavkovik \\ University of Luxembourg \\ marija.slavkovik@uni.lu \\ Leendert van der Torre \\ University of Luxembourg \\ leon.vandertorreduni.lu
}

September 9, 2011

\begin{abstract}
Many voting rules are based on minimization or maximization principle. Likewise, in the field of logic-based knowledge representation and reasoning, many belief change or inconsistency handling operators make use of minimization. Surprisingly, minimization has not played a major role in the field of judgment aggregation, in spite of its proximity to voting theory and logic-based knowledge representation and reasoning. Here we make a first step in the study of judgment aggregation rules based on minimization, and propose a classification of judgment aggregation rules based on some minimization or maximization principle. We distinguish four families. The rules of the first family compute the collective judgment for each issue, using proposition-wise majoritarian aggregation, and then restore consistency using some minimal change principle. The rules of the second family proceed in a similar way but take into account the strength of the majority on each issue. Those of the third family consist in restoring the consistency of the majoritarian judgment by removing or changing some individual judgments in a minimal way. Finally, those of the fourth family are based on some predefined distance between judgment sets, and look for a consistent collective judgment minimizing the overall distance to the individual judgment sets. For each family we propose a few typical rules. While most of these rules are new, a few ones correspond to rules
\end{abstract}

\footnotetext{
*A preliminary version of this paper appeared in the Proceedings of the 13th International Conference on the Theoretical Aspects of Reasoning About Knowledge (TARK-11)[20].
} 
that have been defined elsewhere. We study the inclusion relationships between

these rules and address some of their social choice theoretic properties.

\section{Introduction}

In voting theory and in computational social choice, a large body of work focuses on specific voting rules: how their winner sets compare to each other; their social choicetheoretic properties; the computational and communication complexity of winner determination; the theoretical and experimental study of manipulability and control; the amount of information necessary to determine the outcome; etc.

A judgment aggregation problem is specified by a set of logically related issues, an agenda, on which the agents cast judgments. The judgments are typically Boolean evaluations of the agenda issues. A judgment aggregation rule amalgamates the individual judgments into a collective set of judgments, which should adhere to the logical relations of the agenda issues. Unlike in voting, the bulk of the research in judgment aggregation focuses on possibility and impossibility results: typically, one looks for minimal conditions on the structure of the agenda, on the allowed judgment sets, or on the properties of the logical system, implying the existence or the non-existence of judgment aggregation rules satisfying a small set of desirable properties (such as nondictatorship, unanimity, independence etc.); or else, one looks for a characterization of all judgment aggregation rules satisfying a set of properties, possibly under some domain restrictions. But the focus on specific rules, or families of voting rules, and their properties, has been the topic of few papers. Still, there are a few exceptions, that we list now.

- The premise-based procedure has been introduced in [19] under the name "issueby-issue voting" and studied in $[11,26]$. For this procedure, the agenda is assumed to be partitioned into two subsets: premises and conclusions. The premises are logically independent. The individuals vote on the premises and the majority on each premise is used to find the collective outcome for that premise. From these collective outcomes on the premises, the collective conclusions are derived using either the logical relationships among, or some external constraints regarding the agenda issues. On the other hand, in the conclusion-based procedure, individuals decide privately on the premises and express publicly only their judgments on the conclusions.

- The more general sequential procedures $[22,8,21]$ proceed this way: the elements of the agenda are considered sequentially, following a fixed linear order over the agenda (corresponding for instance to temporal precedence or to priority) and earlier decisions constrain later ones. Collective consistency is guaranteed by definition. Of course, in the general case, the result depends on the choice of the order, i.e. it is path-dependent. Premise-based procedures are specific instances of sequential procedures.

- Quota-based rules $[8,6]$ are a class of rules where each proposition of the agenda is associated with a quota, and the proposition is accepted only if the proportion 
of individuals accepting it is above the quota. For example, uniform rules take the same quota for all elements of the agenda. The majority rule is a special case of quota-based rules. In [8] sequential quota rules are also considered.

- Distance-based rules [25, 29] assume a predefined distance between judgment sets and/or profiles and choose as collective outcome the consistent judgment sets which are closest (for some notion of closeness) to the individual judgments (see Section 3.4).

Even if a few families of judgment aggregation rules have been proposed and studied, still the focus on the research is more on the search for impossibility theorems and axiomatic characterizations of families of rules, which contrasts with voting theory, where voting rules are defined and studied per se.

In voting theory, quite a number of rules are based on some minimization (or maximization) process: for instance, Kemeny, Dodgson, Slater, ranked pairs, maximin etc. (We shall not recall the definition of all these voting rules; the reader can refer, for instance, to [2] for a survey.) Minimization is also a common way of defining reasoning rules (such as belief revision operators, inconsistency handling procedures, or nonmonotonic inference rules) in the community of logic-based knowledge representation and reasoning: typically, one deals with inconsistency by looking for maximal consistent subsets of an inconsistent knowledge base. Belief revision often amounts to incorporating a piece of information to a knowledge base while minimizing the information loss from the initial knowledge base. Similar minimization processes are at work in reasoning about action, belief update and belief merging.

In contrast, with the exception of distance-based rules, minimization has rarely been considered for judgment aggregation. Here we aim at filling this gap by proposing several families of minimization-based rules, and for each family, a few specific rules that we argue to be among the most natural ones. Our rules maximize the portion of a profile we wish to keep. The way such a maximization is defined depends on the specific rule. Thus, the maximization operated by our aggregation rules is equivalent to minimizing the portion of a profile we wish to remove. In other words, we call our rules "based on minimization", but we could as well say that our rules are based on maximization. Most of the rules we introduce here are new, while a few of them correspond, up to some minor details, to judgment aggregation rules already proposed in the literature. We relate them to similar rules in voting theory and/or knowledge representation and reasoning. We study their interrelationships by showing that in most cases, the proposed rules are inclusion-wise incomparable. We also study their links with existing aggregation rules such as sequential or quota-based rules, and some of their social choice theoretic properties (majority-preservation, unanimity, monotonicity, reinforcement).

Aggregation conditions such as anonymity (a permutation on the individual judgment sets does not alter the collective outcome) and neutrality (the elements of the agenda are aggregated in the same way) can be defined as usual (and we omit their formal definitions). It is important to note that none of the rules introduced in this paper satisfies independence (neither do sequential and distance-based rules). For many agendas, the independence condition is sufficient for an impossibility result to occur 
[23]. On the other hand, independence is a key condition to ensure that an aggregation function is non manipulable $[5,7]$. This makes independence an instrumentally attractive condition, as it happens for the independence of the irrelevant alternatives condition in preference aggregation. However, independence has also been severely criticized in the literature (see, for example, $[4,26]$ ) for being incompatible with a framework whose aim is to aggregate logically interrelated propositions.

\section{Judgment aggregation rules}

\subsection{General definitions}

Let $L$ be a propositional language built on a finite set of propositional symbols $P S$. $C n$ denotes logical closure, that is, $\operatorname{Cn}(S)=\{\alpha \in L|S|=\alpha\}$. Lastly, if $S$ is a finite set of formulas of $L$, then $\wedge(S)$ is the conjunction of all formulas in $S$.

\section{Definition 1 (agendas, judgment sets, profiles)}

- an agenda is a finite set $X=\left\{\varphi_{1}, \neg \varphi_{1}, \ldots, \varphi_{m}, \neg \varphi_{m}\right\}$ of propositional formulae of $L$, consisting of pairs of propositions $\varphi_{i}, \neg \varphi_{i}$, and containing neither tautologies not contradictions. The pre-agenda $[X]$ associated with $X$ is $[X]=\left\{\varphi_{1}, \ldots, \varphi_{m}\right\}$. $A$ subagenda of $X$ is a subset of $X$ being itself an agenda, that is, containing pairs of propositions $\varphi, \neg \varphi$, where $\varphi \in[X]$.

- a judgment set over $X$ is a subset of $X$. A judgment set $A$ is complete if for every pair $\{\varphi, \neg \varphi\}$ in $X$, A contains either $\varphi$ or $\neg \varphi$. A judgment set $A$ is consistent if $\bigwedge\left\{\varphi_{j} \mid \varphi_{j} \in A\right\}$ is satisfiable. $J S(X)$ and $C J S(X)$ respectively denote the set of all complete judgment sets, and the set of all consistent and complete judgment sets over $X$.

- an $n$-voter profile over $X$ is a collection $P=\left\langle A_{1}, \ldots, A_{n}\right\rangle$ where each $A_{i}$ is a consistent and complete ${ }^{1}$ judgment set.

We now define judgment aggregation rules. ${ }^{2}$ As in voting theory, we distinguish between deterministic rules, mapping a profile to a single collective judgment set, and nondeterministic rules (or correspondences), mapping a profile to a nonempty set of collective judgment sets.

\section{Definition 2 (judgment aggregation rules)}

- $a$ deterministic judgment aggregation rule is a mapping $f_{n, X}$ associating with every profile $P=\left\langle A_{1}, \ldots, A_{n}\right\rangle$ a consistent judgment set $f_{n, X}(P)$. A deterministic aggregation rule $f_{n, X}$ is complete if for every profile $P, f_{n, X}(P)$ is complete.

\footnotetext{
${ }^{1}$ In judgment aggregation consistent and complete judgment sets are usually assumed. However, while consistency seems an indispensable requirement, completeness can be dismissed, at least in some contexts. Some works $[15,12]$ investigated what happens if we allow voters to abstain from expressing judgments on some propositions in the agenda. We could also define profiles more generally by allowing individual judgment sets to be incomplete. Most of our results would not be altered, but some of the definitions, especially distance-based rules, would be more complicated.

${ }^{2}$ Strictly speaking, a rule is a function; we keep the terminology "rule" for the sake of the parallel with voting theory.
} 
- $a$ nondeterministic judgment aggregation rule (or judgment aggregation correspondence) is a mapping $F_{n, X}$ associating with every profile $P$ a nonempty set of consistent judgment sets $F_{n, X}(P)$.

Most of the time, when referring to judgment aggregation rules we will keep $n$ and $X$ implicit when they are clear from the context, i.e., $f_{n, X}$ (resp. $F_{n, X}$ ) will be simply denoted as $f$ (resp. $F$ ). Also, by a slight abuse of language, if $P=\left\langle A_{1}, \ldots, A_{n}\right\rangle$, then we will write $f\left(A_{1}, \ldots, A_{n}\right)$ and $F\left(A_{1}, \ldots, A_{n}\right)$ instead of $f\left(\left\langle A_{1}, \ldots, A_{n}\right\rangle\right)$ and $F\left(\left\langle A_{1}, \ldots, A_{n}\right\rangle\right)$.

As in voting theory, a rule can be obtained from a correspondence using a tiebreaking mechanism, such as a priority over judgment sets, or over agents. As in voting, we need nondeterminism because of ties - here, ties between judgment sets. For instance, if $X=\{a, \neg a\}$ and $P=\langle a, a, \neg a, \neg a\rangle$ then in order to be anonymous and non-biased, $R(P)$ has to contain the two judgment sets $\{a\}$ and $\{\neg a\}$, that is, $R(P)=\{\{a\},\{\neg a\}\}$. In the rest of the paper we focus on nondeterministic rules, unless we state the contrary ${ }^{3}$.

There are two different views of aggregation rules: either we see the output as a mere collection of consistent judgment sets, or we see it as a closed logical theory.

Definition 3 (logical theory $T_{F}(P)$ )

Given a judgment aggregation rule $F$, and a profile $P$, we define the logical theory $T_{F}(P)=\bigcap\{C n(A) \mid A \in F(P)\}$.

Let $F$ and $F^{\prime}$ be two aggregation rules. $F$ and $F^{\prime}$ are theory equivalent, denoted $F={ }_{T} F^{\prime}$ if for every profile $P$ we have $T_{F}(P)=T_{F^{\prime}}(P)$, (and simply equal, denoted $F=F^{\prime}$, if for every profile $P$ we have that $F(P)=F\left(P^{\prime}\right)$ ). $F$ is at least as discriminant as $F^{\prime}$ if for every profile $P$ we have $T_{F^{\prime}}(P) \subseteq T_{F}(P) . F$ and $F^{\prime}$ are incomparable if there exists two profiles $P$ and $Q$ such that $T_{F}(P) \nsubseteq T_{F^{\prime}}(P)$ and $T_{F}(Q) \nsubseteq T_{F^{\prime}}(Q)$.

Thus, a formula $\alpha$ is in $T_{F}(P)$ if and only if it can be inferred from every judgment set in $F(P)$. Note that $T_{F}(P)$ being the intersection of consistent closed logical theories, it is itself a consistent closed theory.

\section{Definition 4 (majoritarian aggregation)}

The majority aggregation rule $m$ is defined as: for every profile $P, M(P)$ is a singleton judgment set $\{m(P)\}$ such that for every $\psi \in X, m(P)$ contains $\psi$ if and only if a majority of agents have $\psi$ in their judgment set, that is, if and only if $\#\left\{i \mid \psi \in A_{i}\right\}>\frac{n}{2}$. $m(P)$ is called the majoritarian judgment set associated with $P$. Note that if $n$ is odd then $m(P)$ is a complete judgment set.

A profile $P$ is majority-consistent if $m(P)$ is a consistent judgment set. A judgment aggregation rule $F$ is majority-preserving if, for every majority-consistent profile $P$, $F(P)={ }_{T} M(P)$.

Example 1 Consider the pre-agenda $[X]=\{p \wedge r, p \wedge s, q, p \wedge q, t\}$ and a profile $P$ of 17 voters, presented in Table 1.

We obtain $m(P)=\{p \wedge r, p \wedge s, q, \neg(p \wedge q), t\}$ (and $M(P)=\{m(P)\}$ ). $m(P)$ is an inconsistent judgment set, therefore $P$ is not majority-consistent.

\footnotetext{
${ }^{3}$ One may also want to require that not only the output of a judgment aggregation rule is a single judgment set $A$, but that this judgment set is itself complete. Doing this amounts at having another tie-breaking rule which, in case of a tie between $\varphi$ and $\neg \varphi$, specifies how to break it.
} 


\begin{tabular}{r|ccccc} 
Voters & $\{p \wedge r$, & $p \wedge s$, & $q$, & $p \wedge q$, & $t\}$ \\
\hline$A_{1} \times 6$ & + & + & + & + & + \\
$A_{2} \times 4$ & + & + & - & - & + \\
$A_{3} \times 7$ & - & - & + & - & - \\
\hline $\mathrm{m}(\mathrm{P})$ & + & + & + & - & +
\end{tabular}

Table 1: The profile $\mathrm{P}$

We end this Section by defining distances between judgment sets and between profiles. A distance $d$ between judgment sets over $X$ is a function $d: J S(X) \times J S(X) \rightarrow \mathbf{N}$ such that for all $A, B, C \in J S(X)$, (a) $d(A, B)=0$ if and only if $A=B$, (b) $d(A, B)=$ $d(B, A)$ and (c) $d(A, C) \leq d(A, B)+d(B, C)$. A distance function between profiles is defined similarly. Finally, the Hamming distance between judgment sets and between profiles $[25,14]$ is defined as follows.

Definition 5 (Hamming distance between complete judgment sets $d_{H}$ ) Given two complete judgment sets $A$ and $A^{\prime}$ (over the same set of agents and the same agenda), the Hamming distance $d_{H}$ between $A$ and $A^{\prime}$ is defined by

$$
d_{H}\left(A, A^{\prime}\right)=\left|A \backslash A^{\prime}\right|+\left|A^{\prime} \backslash A\right|
$$

Now, the distance between two profiles is the sum of the Hamming distance between their individual judgment sets:

Definition 6 (Hamming distance between profiles $D_{H}$ ) Given two profiles $P=\left\langle A_{1}, \ldots, A_{n}\right\rangle$ and $Q=\left\langle A_{1}^{\prime}, \ldots, A_{n}^{\prime}\right\rangle$, the Hamming distnce between $P$ and $Q$ is defined by

$$
D_{H}(P, Q)=\sum_{i=1}^{n} d_{H}\left(A_{i} A_{i}^{\prime}\right)
$$

\section{Four families of aggregation rules}

We now give four different families of minimization-based judgment aggregation rules.

\subsection{Rules based on the majoritarian judgment set}

\section{Definition 7 (rule based on the majoritarian judgment set)}

A rule $R$ is based on the majoritarian judgment set if there exists a function $f$ mapping every judgment set (consistent or not) to a nonempty set of consistent judgment sets, such that for every profile $P, R(P)=f(m(P))$.

This family can be viewed as the counterpart, for judgment aggregation, of voting rules that are based on the pairwise majority graph (also known as tournament solutions). Being based on the majoritarian judgment set means, in practice, that knowing the majoritarian judgment set of a profile is enough to determine the outcome of the 
rule, or, equivalently, that two profiles $P$ and $Q$ whose majoritarian judgment coincide $(m(P)=m(Q))$ will lead to the same outcome $(R(P)=R(Q))$. We naturally expect these rules to be majority-preserving, which is equivalent to saying that the restriction of $f$ to consistent judgment sets is the identity: if $J$ is consistent, then $f(J)=\{J\}$; such a condition can be seen as the counterpart, for judgment aggregation, of Condorcetconsistency.

When $m(P)$ is not consistent, we look for a minimal way of restoring consistency by removing some elements form the agenda. Given a judgment set $J$, we define the set of consistent subjudgment sets of $J$, denoted by $\operatorname{Cons}(J)$, as $f(J)=\left\{J^{\prime} \subseteq J \mid J^{\prime}\right.$ consistent $\}$. Defining a rule consists in defining a minimality criteria for the set of formulas removed from $J$. There are two obvious choices, consisting in choosing consistent subjudgment sets of $m(P)$ that that are maximal for, respectively, set inclusion or cardinality, which corresponds to the following choices for $f$ :

- $f(J)=\max (\operatorname{Cons}(J), \supseteq)$;

- $f(J)=\max (\operatorname{Cons}(J),||$.

Equivalently, these rules consist in looking for, respectively, a minimal subset or a minimal number of formulas in $X$ to remove such that the profile becomes majorityconsistent. The formal definition we give correspond to this alternative characterization.

In the following we use the abbreviation maxcard for of maximal cardinality.

Definition 8 (maximal subagenda rule $R_{M S A}$ )

Given a profile $P=\left\langle A_{1}, \ldots, A_{n}\right\rangle$ on an agenda $X,[X]$ the pre-agenda associated with $X$, and a sub-preagenda $[Y] \subseteq[X]$, the restriction of $P$ to $Y$ is $P^{\lfloor Y}=\left\langle A_{j} \cap Y, 1 \leq\right.$ $j \leq n\rangle$. Let $M S A(P)$ the set of all maximal sub-preagendas $[Y]$ of $[X]$ (with respect to set inclusion) such that $P^{\downarrow Y}$ is majority-consistent. The maximal subagenda judgment aggregation rule $R_{M S A}$ maps $P$ to $R_{M S A}(P)=\left\{m\left(P^{\downarrow Y}\right) \mid[Y] \in M S A(P)\right\}$.

Example 2 Consider the same agenda and profile as in Example 1. We obtain that

$$
R_{M S A}(P)=\left\{\begin{array}{rrrr}
\{p \wedge r, & p \wedge s, & q, & t\}, \\
\{p \wedge r, & p \wedge s, & \neg(p \wedge q), & t\}, \\
\{q, & \neg(p \wedge q), & t\} &
\end{array}\right\} .
$$

Instead of looking for maximal majority-consistent subagendas with respect to inclusion we may look instead for maxcard majority-consistent subagendas, which leads to the following judgment aggregation rule, which corresponds, up to some minor details and for a specific choice of a distance function, to the endpoint judgment aggregation rule defined in [25].

Definition 9 (maxcard subagenda rule $R_{M C S A}$ )

Let MCSA $(P)$ the set of all maxcard sub-preagendas $[Y]$ of $[X]$ such that $P^{\downarrow Y}$ is majority-consistent. The maxcard subagenda judgment aggregation rule $R_{M C S A}$ maps $P$ to $R_{M C S A}(P)=\left\{m\left(P^{\downarrow Y}\right) \mid[Y] \in M C S A(P)\right\}$. 
Example 3 Consider again the agenda and profile from Example 1. The sub-preagenda $Y$ which gives a majority-consistent $P^{\downarrow Y}$ and is maximal is obtained for either $Y=$ $\{p \wedge r, p \wedge s, q, t\}$ or $Y=\{p \wedge r, p \wedge s, p \wedge q, t\}$. We obtain

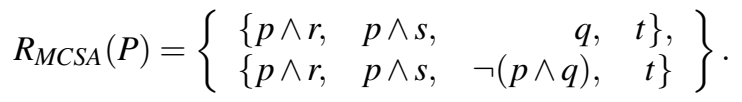

Proposition 1 Let Cons $(m(P))$ be the set of all consistent subsets of $m(P)$.

- $R_{M S A}(P)=\max (\operatorname{Cons}(m(P)), \subseteq)$.

- $R_{M C S A}(P)=\max (\operatorname{Cons}(m(P)),||$.$) .$

Proof: We give the proof for $R_{M S A}$ only; the proof for $R_{M C S A}(P)$ proceeds exactly in the same way.

Let $[Y] \in M S A(P)$. We have $m\left(P^{\downarrow Y}\right) \subseteq m(P)$ and $m\left(P^{\downarrow Y}\right)$ is consistent. Assume that $m\left(P^{\downarrow Y}\right)$ is not a maximal consistent subset of $m(P)$; then there exists a consistent subagenda $Z$ of $X$ such that $m\left(P^{\downarrow Y}\right) \subset Z \subseteq m(P)$. Since both $m\left(P^{\downarrow Y}\right)$ and $Z$ contains at most one of $\varphi, \neg \varphi$ for every $\varphi \in X$ (otherwise they would not be consistent), there must be a $\varphi$ such that either $\varphi \in Z$ or $\neg \varphi \in Z$, and $\varphi \notin[Y]$. But then $[Y] \cup\{\varphi\} \subseteq Z \subseteq m(P)$ and $Z$ consistent implies that $m([Y] \cup\{\varphi\})$ is a consistent subset of $m(P)$, contradicting $[Y] \in M S A(P)$. Therefore, $m\left(P^{\lfloor Y}\right) \in \max (\operatorname{Cons}(m(P)), \subseteq)$.

Conversely, let $Z \in \max (\operatorname{Cons}(m(P)), \subseteq) . \quad Y=\{\varphi \in X \mid \varphi \in Z$ or $\neg \varphi \in Z\}$ is a preagenda of $X$, and because $Z$ is a consistent subset of $m(P), Z$ contains at most one of $\varphi, \neg \varphi$ for every $\varphi \in X$, therefore $m(P)^{\downarrow Y}=Z$. Assume there is a $Y^{\prime} \supset Y$ such that $m\left(P^{\downarrow Y^{\prime}}\right)$ is consistent. Then $m\left(P^{Y^{\prime}}\right) \supset m\left(P^{Y}\right)=Z$, contradicting $Z \in \max (\operatorname{Cons}(m(P)), \subseteq$ ). Therefore, $Y$ is a maximal consistent subpreagenda of $P$.

We note that even when $n$ is odd, $R_{M S A}(P)$ and $R_{M C S A}(P)$ may contain incomplete judgment sets. Take for instance $P=\langle\{a, b, a \wedge b\},\{a, \neg b, \neg(a \wedge b)\},\{\neg a, b, \neg(a \wedge$ $b)\}\rangle$; then $R_{M S A}(P)=R_{M C S A}(P)=\{\{a, b\},\{a, \neg(a \wedge b)\},\{b, \neg(a \wedge b)\}\}$. However, when $n$ is odd, every judgment set in $R_{M S A}(P)$ and a fortiori in $R_{M C S A}(P)$ is equivalent to a complete judgment set: here, $\{a, b\},\{a, \neg(a \wedge b)\}$ and $\{b, \neg(a \wedge b)\}$ are equivalent to, respectively, $\{a, b, a \wedge b\},\{a, \neg b, \neg(a \wedge b)\}$ and $\{\neg a, b, \neg(a \wedge b)\}$. Formally:

Proposition 2 If $n$ is odd then for every $J \in R_{M S A}(P)$ and every $J \in R_{M C S A}(P)$, there is a complete judgment set $J^{\prime}$ such that $J$ is equivalent to $J^{\prime}$.

Proof: Let $J \in R_{M S A}(P)$ and assume $J$ is not equivalent to a complete judgment set; then there is a $\varphi \in X$ such that neither $J \models \varphi$ nor $J \models \neg \varphi$. Because $n$ is odd, $m(P)$ is a complete judgment set, and contains either $\varphi$ or $\neg \varphi$. Without loss of generality, assume it contains $\varphi$. Then $J \cup\{\varphi\} \subseteq m(P)$ and $J \cup\{\varphi\}$ is consistent, contradicting $J \in R_{M S A}(P)$. The proof for $R_{M C S A}$ follows from the fact that $R_{M C S A}(P) \subseteq R_{M S A}(P)$.

While $R_{M S A}$, as far as we know, is new, $R_{M C S A}$ coincides with a rule defined in [25].

Definition 10 (endpoint rule [25]) Let $d$ be a distance function between judgment sets. The judgment aggregation rule Endpoint ${ }_{d}$ is defined by: for any profile $P$,

$$
\text { Endpoint }_{d}(P)=\left\{J \in C J S(X) \mid d(J, m(P)) \leq d\left(J^{\prime}, m(P)\right) \text { for all } J^{\prime} \in C J S(P)\right\}
$$




\section{Proposition $3 R_{M S A}={ }_{T}$ Endpoint $_{d_{H}}$.}

Proof: We claim that for every profile $P$ and every $A \in J S(P)$, we have $A \in M S A(P)$ if and only for every $J \in C J S(X)$ extending $A$ we have $d_{H}(J, m(P)) \leq d_{H}\left(J^{\prime}, m(P)\right)$ for every $J^{\prime} \in C J S(X)$, from which the result follows.

Let $A$ be a consistent subset of $m(P)$ and $J \in C J S(X)$ extending $A$. We have $d_{H}(J, m(P)) \leq m-|A|$. Assume there exists $J^{\prime} \in C J S(X)$ such that $d_{H}\left(J^{\prime}, m(P)\right)<$ $d_{H}(J, m(P)) \leq m-|A|$. Then $\left|J^{\prime} \cap m(P)\right|>|A|$, and $J^{\prime} \cap m(P)$ is a consistent subset of $m(P)$, which implies that $A \notin M S A(P)$. Therefore, for every $J \in C J S(X)$ extending $A$ we have $d_{H}(J, m(P)) \leq d_{H}\left(J^{\prime}, m(P)\right)$ for every $J^{\prime} \in C J S(X)$, which implies that $T_{\text {Endpoint }}(P) \models T_{R_{M C S A}}(P)$.

Conversely, let $J \in C J S(X)$. Let $A=J \cap m(P)$. Then $d_{H}(J, m(P))=m-|A|$ and $A$ is a consistent subset of $m(P)$. Suppose that $A \notin M S A(P)$, then there exists a consistent subset $A^{\prime}$ of $m(P)$ such that $\left|A^{\prime}\right|>|A|$. But now, any $J^{\prime} \in C J S(X)$ extending $A^{\prime}$ is such that $d_{H}\left(J^{\prime}, m(P)\right) \leq m-\left|A^{\prime}\right|<m-|A|=d_{H}(J, m(P))$, which implies that we do not have $d_{H}(J, m(P)) \leq d_{H}\left(J^{\prime}, m(P)\right)$ for every $J^{\prime} \in C J S(X)$. Therefore, $d_{H}(J, m(P)) \leq$ $d_{H}\left(J^{\prime}, m(P)\right)$ for every $J^{\prime} \in C J S(X)$ implies that $J \cap m(P) \in M S A(P)$. This implies that $T_{R_{M C S A}}(P) \models T_{\text {Endpoint }}(P)$.

\subsection{Rules based on the weighted majoritarian judgment set}

We first define the weighted majoritarian judgment set of a profile $P$ as

$$
w(P)=\{\langle\varphi, N(P, \varphi)\rangle, \varphi \in X\}
$$

where $N(P, \varphi)=\#\left\{i, \varphi \in A_{i}\right\}$.

Whereas $m(P)$ keeps only the information about which one, of the two propositions $\varphi$ and $\neg \varphi$, is supported by a majority of voters, $w(P)$ keeps much more information, since it stores the number of voters who support $\varphi$ and $\neg \varphi$. Obviously, $m(P)$ can be recovered from $w(P)$ but not vice versa.

\section{Definition 11 (rule based on the weighted majoritarian judgment set)}

A rule $R$ is based on the weighted majoritarian judgment set if there exists a function $f$ mapping every judgment set (consistent or not) to a nonempty set of consistent judgment sets, such that for every profile $P, R(P)=f(w(P))$.

This family can be viewed as the counterpart, for judgment aggregation, of voting rules that are based on the weighted pairwise majority graph, such as maximin, ranked pairs, or Borda.

The first rule of this class we consider is the maxweight subagenda rule.

$R_{M S A}$ and $R_{M C S A}$ consider the judgments on the agenda subset as a unit that is to be kept in its entirety or got ridden of. A finer way of defining a judgment rule consists in looking for maximal or maxcard majority-consistent subsets of the set of elementary pieces of information consisting each of a pair (element of the agenda, judgment on it elicited from an agent). Equivalently, this comes down to weight each element of the agenda by the number of agents who support it, and then to look for maxweight subagendas. 


\section{Definition 12 (maxweight subagenda rule $R_{M W A}$ )}

For any subagenda $Y \subseteq X$, the weight of $Y$ with respect to $P$ is defined by $w_{P}(Y)=$ $\sum_{\psi \in Y} N(P, \psi)$. Let $M W A(P)$ be the set of all consistent subagendas $Y$ of $X$ maximizing $w_{P}$. The maxweight subagenda judgment aggregation rule $R_{M W A}$ maps $P$ to $R_{M W A}(P)=$ $\{Y \mid Y \in M W A(P)\}$.

Example 4 Consider the agenda and profile of Example 1. We obtain:

$$
\begin{array}{ll}
N(P, p \wedge r)=10, & N(P, \neg(p \wedge r))=7 \\
N(P, p \wedge s)=10, & N(P, \neg(p \wedge s))=7 \\
N(P, q)=13, & N(P, \neg q)=4 \\
N(P, p \wedge q)=6, & N(P, \neg(p \wedge q))=11 \\
N(P, t)=10, & N(P, \neg t)=7
\end{array}
$$

Consequently $R_{M W A}(P)=\{\{p \wedge r, p \wedge s, q, p \wedge q, t\}\}$, because $w_{P}(\{p \wedge r, p \wedge s, q, p \wedge$ $q, t\})=49$ is maximal with respect to all other complete and consistent $Y \subset X$.

The intuition behind this rule is that we look for a minimal number of elementary information items to remove from $P$ so that it becomes majority-consistent, where an information item is an element from $X$ approved by an agent. The set of information items associated with $P$, denoted by $\Sigma(P)$, is the multiset containing as many occurrences of $\varphi$ as agents who approve $\varphi$ in $P$. For instance, if $[X]=\{a, b, c, a \wedge b\}$ and $P=\langle\{a, b, c, a \wedge b\},\{\neg a, b, c, \neg(a \wedge b)\},\{a, \neg b, c, \neg(a \wedge b)\}\rangle$ then $\Sigma(P)=\{a, b, c, a \wedge$ $b, \neg a, b, c, \neg(a \wedge b), a, \neg b, c, \neg(a \wedge b)\}=\{a, a, \neg a, b, b, \neg b, c, c, c, a \wedge b, \neg(a \wedge b), \neg(a \wedge$ b) $\}$.

Now, let MaxCard $(\Sigma(P))$ be the set of all maxcard consistent subsets of $\Sigma(P)$. If $S \in \operatorname{Max} \operatorname{Card}(\Sigma(P))$ then for every $\varphi \in X, S$ contains either all occurrences of $\varphi$ in $\Sigma(P)$ or all occurrences of $\neg \varphi$ in $\Sigma(P)$. Let $J_{S}$ be the judgment set containing $\varphi$ if $S$ contains all occurrences of $\varphi$ in $\Sigma(P)$ and $\neg \varphi$ if $S$ contains all occurrences of $\neg \varphi$ in $\Sigma(P)$. Then $R_{M W A}(P)=\left\{J_{S} \mid \operatorname{Max} \operatorname{Card}(\Sigma(P))\right\}$.

Although it looks entirely new, we will show soon that this natural rule corresponds to a rule already defined, in a totally different way, in [14].See Section 3.4.

Proposition $4 R_{M W A}$ is majority-preserving.

Proof: Let $P$ be a majority-consistent profile. We claim that $R_{M W A}(P)$ consists of all complete consistent subagendas extending $m(P)^{4}$ Let $J$ be a complete consistent subagendas extending $m(P)$. Assume that $J \notin R_{M W A}(P)$ : then there exists a consistent subagenda $J^{\prime}$ such that $w_{P}\left(J^{\prime}\right)>w_{P}(J)$. This implies that there must be a $\varphi \in X$ such that $\varphi \in J, \neg \varphi \in J^{\prime}$, and $N(P, \neg \varphi)>N(P, \varphi)$, which implies that $\varphi \notin m(P)$, which contradicts the assumption that $J$ extends $m(P)$.

The following rule is inspired from the ranked pairs rules in voting theory [31]. It consists in fixing first the truth value for the elements of the agenda with the largest

\footnotetext{
${ }^{4}$ Note that when $n$ is odd, then $m(P)$ is a complete subagenda, so in this case $R_{M W A}(P)=\{m(P)\}$; however, if $n$ is even then $m(P)$ might be incomplete. For instance, if $n=2,[X]=\{p, q\}$ and $P=\langle\{p, q\},\{p, \neg q\}\rangle$ then $m(P)=\{p\}$ and $R_{M W A}(P)=\{\{p, q\},\{p, \neg q\}\}$.
} 
majority, and iterate, considering the elements of the agenda in the decreasing order of the number of agents who support them, and fix their value to the majoritarian value as long as this is possible without producing an inconsistency.

\section{Definition 13 (ranked agenda $R_{R A}$ )}

Let $Y=\left\{\varphi \in X \mid N(P, \varphi)>\frac{n}{2}\right\}$, and let $\geq_{P}$ the complete weak order relation on $Y$ defined by $\varphi \succeq_{P} \psi$ if $N(P, \varphi) \geq N(P, \psi)$. $R_{R A}(P)$ is defined as follows: $A \in R_{R A}(P)$ if there exists a linear order $\succ$ on $X$ refining $\geq$ such that $R A(\succ, P)=A$, where $R A(\succ, P)$ is defined inductively by

- reorder the elements of $Y$ following $\succ$, i.e., such that $\varphi_{\sigma(1)} \succ \ldots \varphi_{\sigma(m)}$;

- $D:=\emptyset$;

- for $k:=1$ to $m$ do: if $D \cup\left\{\varphi_{\sigma(i)}\right\}$ is consistent then $D:=D \cup\left\{\varphi_{\sigma(i)}\right\}$;

- $R A(\succ, P):=D$.

Clearly, $R_{R A}$ is based on the weighted majoritarian judgment set.

Example 5 Take again the same profile as in Example 1. We have $Y=\{p \wedge r, p \wedge$ $s, q, \neg(p \wedge q), t\}$, and $q>_{P} \neg(p \wedge q)>_{P} p \wedge r \sim_{P} p \wedge s \sim_{P} t$ (where $\sim_{P}$ and $>_{P}$ are respectively the indifference and the strict preference relations induced from $\succeq_{P}$ ). We obtain

$$
R_{R A}(P)=\{\{q, \neg(p \wedge q), t, \neg(p \wedge r), \neg(p \wedge s)\}\} .
$$

We note that every judgment set $J$ in $R_{R A}(P)$ is complete; if not, there would be a $\varphi \in X$ such that neither $\varphi$ not $\neg \varphi$ is in $J$; now, since $J$ is consistent, either $J \cup\{\varphi\}$ or $J \cup\{\neg \varphi\}$ is consistent. But then, either $\varphi$ or $\neg \varphi$ would have been incorporated in $J$, which contradicts the assumption that $J$ contains neither $\varphi$ nor $\neg \varphi$. (More generally, when the number of voters $n$ is odd, each of the collective judgment sets obtained from any of the rules introduced so far is equivalent to a complete judgment set.)

Proposition $5 R_{R A}$ is majority-preserving.

Proof: In $\succ$, the elements of $m(P)$ are considered before the elements of $X \backslash m(P)$. Therefore, when an element $\varphi$ of $m(P)$ is considered, the current judgment set $D$ is a subset of $m(P)$ and $D \cup\{\varphi\} \subseteq m(P)$, therefore $D \cup\{\varphi\}$ is consistent, which implies that $\varphi$ is incorporated into $D$. Since this is true for any $\varphi \in X$, we get that any element of $R_{R A}(P)$ contains $m(P)$.

Now, let $J$ be a consistent, complete extension of $m(P)$. Take $\succ$ such that all elements of $m(P)$ are considered first, then all elements of $J \backslash\{m(P\}$, then all elements of $X \backslash J$. This order refines $\succ_{P}$, because if $\varphi \in P m(P)$ then $N(P, \varphi)>\frac{n}{2}$, if $\varphi \in J \backslash\{m(P\}$ then $N(P, \varphi)=\frac{n}{2}$ and if $\varphi \in X \backslash J$ then $N(P, \varphi) \leq \frac{n}{2}$. Lastly, $R A(\succ, P)=J$, which proves that $J \in R_{R A}(P)$. 


\subsection{Rules based on the removal or change of individual judgments}

The principle at work, for this family, is that we look for a modified profile, as close as possible to the original profile (with respect to a given distance), such that the resulting profile is majority-consistent. Different rules will be obtained with different distance functions.

This family can be viewed as the counterpart, for judgment aggregation, of voting rules that are based on performing minimal operations on profiles so as to obtain a profile for which there is a Condorcet winner, such as, typically, the Young and Dodgson rules. (See [13] for a general family of voting rules of that kind).

Unsurprisingly, the first rule we consider is called the Young rule for judgment aggregation, by analogy with the Young rule in voting, which outputs the candidate $x$ minimizing the number of voters to remove from the profile so that $x$ becomes a Condorcet winner.

\section{Definition 14 (Young rule for judgment aggregation $R_{Y}$ )}

Given a profile $P=\left\langle A_{1}, \ldots, A_{n}\right\rangle$ and a subset of agents $J \subseteq\{1, \ldots, n\}$, the restriction of $P$ to $J$ is $P_{J}=\left\langle A_{j}, j \in J\right\rangle$, and is called a subprofile of $P$. Let $M S P(P)$ be the set of maxcard majority-consistent subprofiles of $P$ for which $M\left(P_{J}\right)$ is a complete judgment set. Then the Young judgment aggregation rule $Y$ maps $P$ to $R_{Y}(P)=\left\{m\left(P_{J}\right) \mid\right.$ $\left.P_{J} \in \operatorname{MSP}(P)\right\}$.

Intuitively, this rule consists in removing a minimal number of agents so that the profile becomes majority-consistent. Or, equivalently, we maximize the number of voters we keep of a given profile. Obviously, if the profile $P$ is majority-consistent, then no voter needs to be removed and $Y(P)=\{m(P)\}$, hence $Y$ is majority-preserving.

Example 6 Once again we consider $P$ for $X$ given in Example 1. The result

$$
R_{Y}(P)=\{\{\neg(p \wedge r), \neg(p \wedge s), q, \neg(p \wedge q)\}\}
$$

is obtained by removing 3 of the judgment sets $\{p \wedge r, p \wedge s, q,(p \wedge q), t\}$. Removing less, or other 3 judgment sets, does not lead to a majority-consistent profile.

Instead of looking for a minimal number of individual judgments to remove, we can look for a minimal set of individual judgments to remove, leading to an inclusionbased version $R_{I Y}$ of the Young judgment aggregation rule. $R_{I Y}$ is obviously majoritypreserving, for the same reason as $R_{Y}$. Another possibility consists in enlarge-ing the profile (instead of reducing it) by duplicating a minimal number of judgment sets in it so as to make it majority-consistent; we get a rule that we call the reversed Young judgment aggregation rule $R_{R Y}$; it is also obviously majority-preserving.

The last rule we define does not remove agenda elements and/or voters, but looks for a minimal number of atomic changes in the profile so that $P$ becomes majorityconsistent, where an atomic change is the change of truth value of one element of the preagenda in an individual judgment set. For instance, if $A_{1}=\{p, q, p \wedge q, r, p \wedge r\}$, then $A_{1}^{\prime}=\{\neg p, q, \neg(p \wedge q), r, \neg(p \wedge r)\}$ is obtained from $A_{1}$ by a series of three atomic changes (change in the truth value of $p$, of $p \wedge q$ and of $p \wedge r$ ). 


\begin{tabular}{r|ccccc} 
Voters & $p \wedge r$ & $p \wedge s$ & $q$ & $p \wedge q$ & $t$ \\
\hline $6 \times$ & + & + & + & + & + \\
$4 \times$ & + & + & - & - & + \\
$3 \times$ & - & - & + & + & - \\
$4 \times$ & - & - & + & - & - \\
\hline$m(Q)$ & + & + & + & + & +
\end{tabular}

Table 2: The profile $Q$.

This rule is, in spirit, close to Dodgson's voting rule, which looks for the smallest number of elementary changes in a profile so as to make it possess a Condorcet winner. Replacing having a Condorcet winner by being majority-consistent and adapting the notion of elementary change, we get our judgment aggregation rule.

Definition 15 (minimal number of atomic changes rule $R_{M N A C}$ ) Given a profile $P, a$ profile $Q$ consisting of complete and consistent individual judgment sets is a closest majority-consistent profile to $P$ if $Q$ is majority-consistent, and there is no majorityconsistent profile $Q^{\prime}$ such that $D_{H}\left(P, Q^{\prime}\right)<D_{H}(P, Q)$. Let $C M C(P)$ the set of all closest majority-consistent profile to $P$. The minimal number of atomic changes rule is defined by

$$
R_{M N A C}(P)=\{m(Q) \mid Q \in C M C(P)\}
$$

$R_{M N A C}$ is not a new rule. Full, one of the four methods introduced by Miller and Osherson [25], looks for the closest profile of individual judgments that yields a consistent proposition-wise majority output, and then take this output. Therefore, $R_{M N A C}$ corresponds to the Full voting rule together with the choice of the Hamming distance (while Miller and Osherson do not commit to a specific distance metric) $)^{5}$

Example 7 Consider the profile $P$ from Example 1. The profile $Q$ given on Table 2 is the closest majority-consistent profile to $P$ with $D(P, Q)=3$.

We obtain $R_{M N A C}(P)=\{p \wedge r, p \wedge s, q, p \wedge q, t\}$.

Clearly, if $P$ is majority-consistent then no elementary change is needed, therefore $R_{M N A C}$ is majority-preserving.

Now, $R_{M N A C}$ is not a new rule. Full, one of the four methods introduced by Miller and Osherson [25], looks for the closest profile of individual judgments that yields a consistent proposition-wise majority output, and then take this output. The difference between our minimal number of atomic changes rule and Full is that we use to Hamming distance, while Miller and Osherson do not commit to a specific distance metric.

Of course, we could also look for the closest profiles $Q$ with respect to set inclusion. But this would give a very weak rule $R$ where $\varphi$ belongs to some judgment set of $R(P)$ as soon as one individual judgment contains $\varphi$.

\footnotetext{
${ }^{5}$ Another possible choice would consist in allowing the modified profile to be individually inconsistent, leading to the so-called Output rule in [25].
} 


\subsection{Distance-based rules}

Two classes of distance-based rules have been used in judgment aggregation, the first one characterised by the minimization of distances between judgment sets [29, 14, 25], derived from distance-based merging operators for belief bases [17, 16], and the second one characterised by the minimization of distances between profiles [25]. The rules we consider in this section resort to some kind of minimization of distances between judgment sets. On the other hand, the minimal number of atomic changes rule $\left(R_{M N A C}\right)$ that we introduced in the previous section is in the spirit of the minimally changing a profile in order to obtain a majority-consistent collective judgment set as in [25].

In [25] four distance-based rules for judgment aggregation are proposed. We have already discussed three of them, namely Full, Output and Endpoint. The fourth one, Prototype, is defined as follows.

Definition 16 Prototype $\left(A_{1}, \ldots, A_{n}\right)$ is the set of all judgment sets $A$ in $\operatorname{CJS}(X)$ such that $\left.\sum_{i=1}^{n} d\left(A, A_{i}\right) \leq \sum_{i=1}^{n} d\left(A^{\prime}, A_{i}\right), \forall A^{\prime} \in \Phi_{x}\right\}$.

This rule has also been considered independently in [14].

We now propose a larger family of aggregation rule, in the same sprit as [25].

Let $d: \Phi_{X} \times \Phi_{X} \mapsto \mathbb{R}^{+}$be a distance function between judgment sets from $\Phi_{X}$ and $\odot:\left(\mathbb{R}^{+}\right)^{n} \mapsto \mathbb{R}^{+}$be a symmetric, non-decreasing aggregation function such that, for every $x, y, x_{1}, \ldots, x_{n} \in \mathbb{R}$, has the following properties: $\odot(x, \ldots, x)=x ; \odot\left(x_{1}, \ldots, x_{n}\right)=$ 0 if and only if $x_{1}=\ldots=x_{n}=0$.

The distance-based judgment aggregation rule $R^{d, \odot}$ induced by $d$ and $\odot$ is defined by:

$$
R^{d, \odot}\left(A_{1}, \ldots, A_{n}\right)=\underset{A \in \Phi_{X}}{\arg \min } \odot\left(d\left(A, A_{1}\right), \ldots d\left(A, A_{n}\right)\right) .
$$

Definition 17 A judgment aggregation rule is distance-based if it is equal to $R^{d, \odot}$ for some $d$ and $\odot$.

Here we consider only $\odot=\sum$ and $\odot=\max$, and the Hamming distance $d_{H}{ }^{6}$ on complete judgment sets, defined as

$$
d_{H}\left(A, A^{\prime}\right)=\left|A \backslash A^{\prime}\right|+\left|A^{\prime} \backslash A\right| .
$$

In the case when $\odot=\sum$ we obtain the distance-based procedure of [14].

We choose $R^{d_{H}, \Sigma}$ and $R^{d_{H}, \max }$ because they capture respectively the intuition of a majoritarian operator and of compromise between the individuals' judgments [3, 18]. Whereas the minimization of the sum of the distances is equivalent to propositionwise majority voting, the minimization of the maximum distance minimizes the disagreement with the least satisfied individual, hence guaranteeing some degree of compromise.

The first result shows that $R^{d_{H}, \Sigma}$ and $R_{M W A}$ are equivalent.

\footnotetext{
${ }^{6}$ We could also consider the "drastic distance" $d_{D}$, defined as $d_{D}\left(A, A^{\prime}\right)=0$ if and only if $A=A^{\prime}$ and $d_{D}\left(A, A^{\prime}\right)=1$ otherwise. Taking $d=d_{D}$ and $\odot=\Sigma$ leads to a judgment aggregation rule that selects the judgment sets given by the highest number of agents, while taking $\odot=\max$ also leads to a rule of no interest.
} 
Proposition $6 R^{d_{H}, \Sigma}$ and $R_{M W A}$ are classically equivalent.

Proof: Given two complete judgment sets $A$ and $A^{\prime}$, and $\varphi \in X$, define $h\left(\varphi, A, A^{\prime}\right)=$ 1 if $\varphi \in\left(A \backslash A^{\prime}\right) \cup\left(A^{\prime} \backslash A\right)$ and $h\left(\varphi, A, A^{\prime}\right)=0$ otherwise.

Now, for any profile $P=\left\langle A_{1}, \ldots, A_{n}\right\rangle$ and any complete judgment set $A$, we have

$$
\begin{aligned}
& \sum_{i=1}^{n} d_{H}\left(A, A_{i}\right) \\
= & \sum_{i=1}^{n} \sum_{\varphi \in X} h\left(\varphi, A, A_{i}\right) \\
= & \sum_{i=1}^{n}\left(\sum_{\varphi \in A} h\left(\varphi, A, A_{i}\right)+\sum_{\varphi \notin A} h\left(\varphi, A, A_{i}\right)\right) \\
= & \sum_{i=1}^{n}\left(\sum_{\varphi \in A} h\left(\varphi, A, A_{i}\right)+\sum_{\neg \varphi \in A} h\left(\varphi, A, A_{i}\right)\right) \\
= & \sum_{i=1}^{n}\left(\sum_{\varphi \in A} h\left(\varphi, A, A_{i}\right)+\sum_{\varphi \in A} h\left(\neg \varphi, A, A_{i}\right)\right) \\
= & \sum_{\varphi \in A}\left(\sum_{i=1}^{n} h\left(\varphi, A, A_{i}\right)+\sum_{i=1}^{n} h\left(\neg \varphi, A, A_{i}\right)\right) \\
= & \sum_{\varphi \in A}(n-N(P, \varphi)+N(P, \neg \varphi)) \\
= & \sum_{\varphi \in A} 2(n-N(P, \varphi)) \\
= & 2 n *|A|-2 w_{P}(A)
\end{aligned}
$$

Therefore, $\sum_{i=1}^{n} d_{H}\left(A, A_{i}\right)$ is minimum if and only if $A \in M W A(P)$, that is, $w_{P}(A)$ is maximum. Since every element of $M W A(P)$ is a complete judgment set, $M W A(P)$ is equal to the set of all complete judgment sets minimizing $\sum_{i=1}^{n} d_{H}\left(A, A_{i}\right)$, which allows us to conclude that $R^{d_{H}, \Sigma}$ and $R_{W M A}$ are equivalent.

Comparing the Definition 17 and the definition Prototype ${ }_{d}$, as it was indicated in [25], we observe that for all profiles $P, R^{d_{H}, \Sigma}(P)=$ Prototype $_{d_{H}}(P)$. Consequently also, for all profiles $P, R_{M W A}(P)=\operatorname{Prototype}_{d_{H}}(P)$.

As a consequence, $R^{d_{D}, \Sigma}$ is majority-preserving. This is however not the case for $R^{d_{D}, \text { max }}$, which is the only one of our rules failing to satisfy majority-preservation.

Proposition $7 R^{d_{H}, \max }$ is not majority-preserving.

Proof: Consider the agenda $X=\{a, \neg a, b, \neg b\}$ and $P=\langle\{a, b\},\{a, b\},\{\neg a, \neg b\}\rangle$. Then $R^{d_{H}, \max }(P)=\{\{a, \neg b\},\{\neg a, b\}\}$; however, $P$ is majority-consistent and $M(P)=$ $\{\{a, b\}\}$.

Example 8 Consider the profile $P$ for agenda $X$ of Example 1. We obtain that $R^{d_{H}, \Sigma}=$ $\{\{p \wedge r, p \wedge s, q, p \wedge q, t\}\}$ while

$$
R^{d_{H}, \max }(P)=\left\{\begin{array}{rrrrr}
\{\neg(p \wedge r), & \neg(p \wedge s), & q, & \neg(p \wedge q), & t\}, \\
\{\neg(p \wedge r), & p \wedge s, & \neg q, & \neg(p \wedge q), & t\}, \\
\{\neg(p \wedge r), & p \wedge s, & q, & p \wedge q, & t\}, \\
\{p \wedge r, & \neg(p \wedge s), & \neg q, & \neg(p \wedge q), & t\}, \\
\{p \wedge r, & \neg(p \wedge s), & q, & p \wedge q, & t\}, \\
\{p \wedge r, & p \wedge s, & \neg q, & \neg(p \wedge q), & \neg t\}, \\
\{p \wedge r, & p \wedge s, & \neg q, & p \wedge q, & \neg t\}
\end{array}\right\} .
$$

The full calcuations are presented in Table 3. 


\begin{tabular}{|c||ccccc|c|c|c||c|c|}
\hline$A_{i}$ & $\{p \wedge r$, & $p \wedge s$, & $q$, & $p \wedge q$, & $t\}$ & $d_{H}\left(A, A_{20}\right)$ & $d_{H}\left(A_{i}, A_{18}\right)$ & $d_{H}\left(A_{i}, A_{3}\right)$ & $\sum$ & $\max$ \\
\hline$A_{1}$ & - & - & - & - & - & 5 & 3 & 1 & 49 & 5 \\
$A_{2}$ & - & - & - & - & + & 4 & 2 & 2 & 46 & 4 \\
$A_{3}$ & - & - & + & - & - & 4 & 4 & 0 & 40 & 4 \\
$A_{4}$ & - & - & + & - & + & 3 & 3 & 1 & 37 & 3 \\
$A_{5}$ & - & - & + & + & - & 3 & 5 & 1 & 45 & 5 \\
$A_{6}$ & - & - & + & + & + & 2 & 4 & 2 & 42 & 4 \\
$A_{7}$ & - & + & - & - & - & 4 & 2 & 2 & 46 & 4 \\
$A_{8}$ & - & - & + & + & + & 2 & 4 & 2 & 42 & 4 \\
$A_{9}$ & - & + & - & - & - & 4 & 2 & 2 & 46 & 4 \\
$A_{10}$ & - & + & - & - & + & 3 & 1 & 3 & 43 & 3 \\
$A_{11}$ & - & + & + & + & - & 2 & 4 & 2 & 42 & 4 \\
$A_{12}$ & - & + & + & + & + & 1 & 3 & 3 & 39 & 3 \\
$A_{13}$ & + & - & - & - & - & 4 & 2 & 2 & 46 & 4 \\
$A_{14}$ & + & - & - & - & + & 3 & 1 & 3 & 43 & 3 \\
$A_{15}$ & + & - & + & + & - & 2 & 4 & 2 & 42 & 4 \\
$A_{16}$ & + & - & + & + & + & 1 & 3 & 3 & 39 & 3 \\
$A_{17}$ & + & + & - & - & - & 3 & 1 & 3 & 42 & 3 \\
$A_{18}$ & + & + & - & - & + & 2 & 0 & 4 & 40 & 4 \\
$A_{19}$ & + & + & + & + & - & 1 & 3 & 3 & 39 & 3 \\
$A_{20}$ & + & + & + & + & + & 0 & 2 & 4 & 36 & 4 \\
\hline
\end{tabular}

Table 3: The calculations for $R^{d_{H}, \Sigma}(P)$ and $R^{d_{H}, \max }(P)$. Recall that $\sum_{i=j}^{n} d\left(A_{i}, A_{j}\right)=$ $6 d_{H}\left(A_{i}, A_{20}\right)+4 d_{H}\left(A_{i}, A_{18}\right)+7 d_{H}\left(A_{i}, A_{3}\right)$

\section{4 (Non)inclusion relationships between the rules}

In this section we consider the equality and inclusion relationships between the rules we have introduced.

Proposition 8 We have the following diagram (Table 7), where inc means "inclusionwise incomparable", $\subset$ means that $T_{R_{1}}(P) \subset T_{R_{2}}(P)$, for all profiles $P$ where $R_{1}$ is the row rule and $R_{2}$ is the column rule, correspondingly for $\supset$. The number next to inc or $\subset$ denotes the proposition in which the relationship is proved.

\begin{tabular}{|c|c|c|c|c|c|c|}
\hline & $R_{M C S A}$ & $R_{M W A}$ & $R_{R A}$ & $R_{Y}$ & $R_{M N A C}$ & $R^{d_{H}, \max }$ \\
\hline$R_{M S A}$ & $\subset, 9$ & $\subset, 10$ & $\subset, 11$ & inc, 13 & inc, 20 & inc, 12 \\
\hline$R_{M C S A}$ & & inc, 14 & inc, 15 & inc, 13 & inc, 19 & inc, 12 \\
\hline$R_{M W A}$ & & & inc, 18 & inc, 16 & inc, 21 & inc, 12 \\
\hline$R_{R A}$ & & & & inc, 17 & inc, 21 & inc, 12 \\
\hline$R_{Y}$ & & & & & inc, 21 & inc, 12 \\
\hline$R_{M N A C}$ & & & & & & inc, 12 \\
\hline
\end{tabular}

Table 4: A summary of the (non)inclusion relationships between the proposed rules.

We give proofs for each table entry, left to right, top to bottom.

Proposition 9 For all profiles $P, T_{R_{M S A}}(P) \subset T_{R_{M C S A}}(P)$. 
Proof: If $Y \subset[X]$ is a maxcard consistent sub-preagenda (w.r.t. $P$ ) of $[X]$ then it is also a maximal consistent sub-preagenda (w.r.t. $P$ ). Now, if $\alpha \in T_{R_{M S A}}(P)$, then $\alpha$ is inferred in every maximal consistent sub-preagenda, and a fortiori in every maxcard consistent sub-preagenda, therefore $\alpha \in T_{R_{M C S A}}(P)$.

To show that $T_{R_{M C S A}}(P) \nsubseteq T_{R_{M S A}}(P)$, consider the profile $P$ in Example 1. As it can be observed in Example 3, $T_{R_{M C S A}}(P) \models p \wedge r$, but we can observe from Example 2 that $T_{R_{M S A}}(P) \not \models p \wedge r$.

Proposition 10 For all profiles $P, T_{R_{M S A}}(P) \subset T_{R_{M W A}}(P)$.

Proof: If $Y \subset[X]$ is a consistent sub-preagenda maximizing $w_{P}(Y)$, then $m\left(P^{\downarrow Y}\right)$ is a maximal consistent subagenda (w.r.t. $P$ ). Now, if $\alpha \in T_{R_{M S A}}(P)$, then $\alpha$ is inferred in every maximal consistent sub-preagenda, and a fortiori in every maxweight consistent subagenda, therefore $\alpha \in T_{R_{M W A}}(P)$.

To show that $T_{R_{M W A}}(P) \nsubseteq T_{R_{M S A}}(P)$, consider the profile $P$ in Example 1. As it can be observed in Example 4, $T_{R_{M W A}}(P) \models q$, but we can observe from Example 2 that $T_{R_{M S A}}(P) \not \models q$.

Proposition 11 For all profiles $P, T_{R_{M S A}}(P) \subset T_{R_{R A}}(P)$.

Proof: In the construction of $R_{R A}(P)$, let $Z$ be the subset of $X$ composed of the $\psi_{k}$ such that $\delta \wedge \psi_{k}$ is consistent. $Z$ is a maximal consistent subagenda w.r.t. $P$ (it is consistent by construction, and maximal because every time a formula $\psi_{k}$ is rejected, it is because it produces an inconsistency with the formulas already present in $\delta$ ). Now, if $\alpha \in T_{R_{M S A}}(P)$, then $\alpha$ is inferred in every maximal consistent subagenda, and a fortiori in $Z$, therefore $\alpha \in T_{R_{R A}}(P)$.

To show that $T_{R_{R A}}(P) \nsubseteq T_{R_{M S A}}(P)$, consider the profile $P$ in Example 1. As it can be observed in Example 5, $T_{R_{R A}}(P) \models q$, but we can observe from Example 2 that $T_{R_{M S A}}(P) \not \models q$.

Proposition $12 R^{d_{H}, \max }$ is incomparable with all the other rules.

Proof: Let $R$ be a majority-preserving rule. Take the profile $P$ as in the proof of Proposition 7. Then $a \leftrightarrow \neg b \in T_{R^{d} H \text {, max }}(P)$, whereas $a \leftrightarrow \neg b \notin T_{R}(P)$ (since $a \leftrightarrow b \in$ $T_{R}(P)$ ); and $a \in T_{R}(P)$, whereas $a \notin T_{R^{d_{H}} \text {, } \max }(P)$. Therefore, $R^{d_{H}, \text { max }}$ is incomparable with all of the other rules.

Proposition $13 R_{Y}$ is incomparable with $R_{M S A}$ and $R_{M C S A}$.

Proof: Consider the profile $P$ on Table 5, with pre-agenda $[X]=\{a, a \rightarrow(b \vee c), b, c, a \rightarrow$ $(d \vee e), d, e\}$, and three agents with the following information sets: The majoritarian aggregation obtained from this profile is $B=\{a, a \rightarrow(b \vee c), \neg b, \neg c, a \rightarrow(d \vee e), \neg d, \neg e\}$. The minimal inconsistent subsets of $B$ are $\{a, a \rightarrow(b \vee c), b, c\}$ and $\{a, a \rightarrow(d \vee$ $e), d, e\}$, therefore, there $B$ has 10 maximal consistent subsets: 9 containing $a$, two of the three formulas $\{a \rightarrow(b \vee c), \neg b, \neg c\}$ and two of the three formulas $\{a \rightarrow(d \vee$ $e), \neg d, \neg e\}$, and one equal to $B \backslash\{a\}$. These 10 maximal consistent subsets correspond 


$\begin{array}{ccccccccc}a & a \rightarrow(b \vee c) & b & c & a \rightarrow(d \vee e) & d & e \\ + & + & + & - & + & + & - \\ + & + & - & + & + & - & + \\ + & - & - & - & - & - & -\end{array}$

Table 5: The profile $P$.

to 10 maximal subagendas; the only maxcard consistent subagenda is $B \backslash\{a\}$, and in this subagenda of $B, \neg a$ is inferred. Therefore, $T_{R_{M C S A}}(P) \models \neg a$. Now, all sub-profiles of $P$ of size two is majority-consistent, and each of them accepts $a$, therefore $T_{R_{Y}}(P) \models$ $a$. Therefore, $R_{Y}$ and $R_{M C S A}$ are incomparable. For $T_{R_{Y}}(P) \nsubseteq T_{R_{M S A}}(P)$, take the same profile as above and note that $a \in T_{R_{Y}}(P)$ but $a \notin T_{R_{M S A}}(P)$. For $T_{R_{M S A}}(P) \nsubseteq T_{R_{Y}}(P)$, assume the pre-agenda is extended with another agenda item $f$, on which the agents vote,,++- . We have $f \in T_{R_{M S A}}$ but $f \notin T_{R_{Y}}$.

Proposition $14 R_{M W A}$ is incomparable with $R_{M C S A}$.

Proof: Take the following seven agent profile $P$ :

\begin{tabular}{c|ccc} 
& $a$ & $b$ & $a \wedge b$ \\
\hline $3 \times$ & + & + & + \\
$2 \times$ & + & - & - \\
$2 \times$ & - & + & -
\end{tabular}

We obtain that $R_{M W A}(P)=\{\{a, b, a \wedge b\}\}$, while $R_{M C S A}(P)=\{\{a, b\},\{a, \neg a \vee \neg b\},\{b, \neg a \vee$ $\neg b\}$. Thus $a \in T_{R_{M W A}}(P)$ whereas $a \notin T_{R_{M C S A}}(P)$. For the converse, in the example of Proposition 13, we have $\neg a \notin T_{R_{M W A}}(P)$ and $\neg a \in T_{R_{M C S A}}(P)$.

Proposition $15 R_{R A}$ is incomparable with $R_{M C S A}$.

Proof: Same profile $P$ as in Proposition 13. We have that $T_{R_{R A}}(P) \models a$. Hence $a \in$ $T_{R_{R A}}(P)$ whereas $\neg a \in R_{M C S A}(P)$, see Proposition 13 .

Proposition $16 R_{M W A}$ is incomparable with $R_{Y}$.

Proof: Consider the following pre-agenda $[X]=\left\{a, a \rightarrow p_{1}, a \rightarrow q_{1}, a \rightarrow\left(p_{1} \wedge q_{1}\right), a \rightarrow p_{2}, a \rightarrow q_{2}, a \rightarrow\left(p_{2} \wedge q_{2}\right), a \rightarrow p_{3}, a \rightarrow\right.$ $\left.q_{3}, a \rightarrow\left(p_{3} \wedge q_{3}\right), a \rightarrow p_{4}, a \rightarrow q_{4}, a \rightarrow\left(p_{4} \wedge q_{4}\right)\right\}$.

Let the profile $P$ be as given on Table 6 .

We obtain that $R_{M W A}(P)=\left\{\left\{\neg a, a \rightarrow p_{1}, a \rightarrow q_{1}, \neg\left(a \rightarrow\left(p_{1} \wedge q_{1}\right)\right), a \rightarrow p_{2}, a \rightarrow\right.\right.$ $q_{2}, \neg\left(a \rightarrow\left(p_{2} \wedge q_{2}\right)\right), a \rightarrow p_{3}, a \rightarrow q_{3}, \neg\left(a \rightarrow\left(p_{3} \wedge q_{3}\right)\right), a \rightarrow p_{4}, a \rightarrow q_{4}, \neg\left(a \rightarrow\left(p_{4} \wedge\right.\right.$ $\left.\left.\left.q_{4}\right)\right)\right\}$ \}. Hence $T_{R_{M W A}}(P) \models \neg a$.

The result for $R_{Y}(P)$ is obtained when exactly one, either one, of the voters is removed. For $R_{Y}(P)$ we obtain $T_{R_{Y}}(P) \models a$.

Proposition $17 R_{R A}$ is incomparable with $R_{Y}$. 


\begin{tabular}{|c|c|c|c|c|c|}
\hline \multirow[b]{2}{*}{ Agenda } & \multicolumn{3}{|c|}{ Voters } & \multirow[b]{2}{*}{$m(P)$} & \multirow[b]{2}{*}{$N\left(P, \varphi_{i}\right)$} \\
\hline & $\times 1$ & $\times 1$ & $\times 1$ & & \\
\hline$a$ & + & + & + & + & 3 \\
\hline$a \rightarrow p_{1}$ & + & + & - & + & 2 \\
\hline$a \rightarrow q_{1}$ & + & - & + & + & 2 \\
\hline$a \rightarrow\left(p_{1} \wedge q_{1}\right)$ & + & - & - & - & 1 \\
\hline$a \rightarrow p_{2}$ & + & + & - & + & 2 \\
\hline$a \rightarrow q_{2}$ & + & - & + & + & 2 \\
\hline$a \rightarrow\left(p_{2} \wedge q_{2}\right)$ & + & - & - & - & 1 \\
\hline$a \rightarrow p_{3}$ & + & + & - & + & 2 \\
\hline$a \rightarrow q_{3}$ & + & - & + & + & 2 \\
\hline$a \rightarrow\left(p_{3} \wedge q_{3}\right)$ & + & - & - & - & 1 \\
\hline$a \rightarrow p_{4}$ & + & + & - & + & 2 \\
\hline$a \rightarrow q_{4}$ & + & - & + & + & 2 \\
\hline$a \rightarrow\left(p_{4} \wedge q_{4}\right)$ & + & - & - & - & 1 \\
\hline
\end{tabular}

Table 6: The profile $P$. The judgment sets are the second, third and fourth column of the table.

Proof: We do not have $T_{R_{R A}}(P) \subseteq T_{R_{Y}}(P)$ as a consequence of Proposition 11 and 13 . For the converse, consider the following profile $P$, with pre-agenda $[X]=\{p, q, p \wedge$ $q, r, s, r \wedge s, t\}$ and 18 agents into six different groups:

\begin{tabular}{c|ccccccc} 
& $p$ & $q$ & $p \wedge q$ & $r$ & $s$ & $r \wedge s$ & $t$ \\
\hline $1 \times$ & + & + & + & - & + & - & + \\
$3 \times$ & + & + & + & - & + & - & - \\
$4 \times$ & + & + & + & + & - & - & - \\
$2 \times$ & + & - & - & + & - & - & - \\
$4 \times$ & + & - & - & + & + & + & + \\
$4 \times$ & - & + & - & + & + & + & +
\end{tabular}

We easily check that the minimal number of agents to remove so as to make the profile majority-consistent is two, and that these agents are the two agents of the fourth group. Therefore, $t \in T_{R_{Y}}(P)$, whereas $t \notin T_{R_{R A}}(P)$.

Proposition $18 R_{R A}$ is incomparable with $R_{M W A}$.

Proof: Same profile as in Proposition 16. We have $\phi_{13} \in T_{R_{R A}}(P)$, whereas $\neg \phi_{13} \in$ $T_{R_{M W A}}(P)$.

Proposition $19 R_{M N A C}$ is incomparable with $R_{M C S A}$.

Proof:To show that there exists a profile $P$ such that $T_{R_{M C S A}}(P) \not \subset T_{R_{M N A C}}$, consider the pre-agenda and profile in the proof of Proposition 13 in which we have that $T_{R_{M C S A}}(P) \models$ $\neg a$. There are 23 profiles $Q$ at a minimal distance $D(P, Q)=2$. We obtain $T_{R_{M N A C}}(P) \not \models$ 


\begin{tabular}{c|ccccccccc} 
Voters & $p$ & $q$ & $p \wedge q$ & $p \wedge \neg q$ & $\alpha_{1}$ & $\alpha_{2}$ & $q \wedge \neg p$ & $\alpha_{3}$ & $\alpha_{4}$ \\
\hline $1 \times$ & + & + & + & - & - & - & - & - & - \\
$1 \times$ & + & - & - & + & + & + & - & - & - \\
$1 \times$ & - & + & - & - & - & - & + & + & + \\
\hline$m(P)$ & + & + & - & - & - & - & - & - & -
\end{tabular}

Table 7: The profile $P$, counterexample for $T_{R_{M S A}}(P) \nsubseteq T_{R_{M N A C}}(P)$.

$\neg a$ because

$$
\begin{aligned}
& R_{M N A C}(P)=\{\quad\{a, a \rightarrow(b \vee c), \neg b, c, a \rightarrow(d \vee e), \neg d, e\}, \\
& \{a, a \rightarrow(b \vee c), \neg b, c, a \rightarrow(d \vee e), d, \neg e\}, \\
& \{a, a \rightarrow(b \vee c), b, \neg c, a \rightarrow(d \vee e), \neg d, e\}, \\
& \{a, a \rightarrow(b \vee c), b, \neg c, a \rightarrow(d \vee e), d, \neg e\}, \\
& \{a, \neg(a \rightarrow(b \vee c)), \neg b, \neg c, \neg(a \rightarrow(d \vee e)), \neg d, \neg e\} \\
& \{\neg a, a \rightarrow(b \vee c), \neg b, \neg c, a \rightarrow(d \vee e), \neg d, \neg e\}\} .
\end{aligned}
$$

To show that that there exists a profile $P$ such that $T_{R_{M N A C}} \not \subset T_{R_{M C S A}}(P)$, consider the profile $P$ from Example 1. We have $T_{R_{M N A C}}(P) \models q$, see Example 7, but $T_{R_{M C S A}}(P) \not \models q$, see Example 3.

Proposition $20 R_{M N A C}$ is incomparable with $R_{M S A}$.

Proof: To show that there exists a $P$ such that $T_{R_{M S A}}(P) \nsubseteq T_{R_{M N A C}}(P)$, consider the pre-agenda $[X]=\left\{p, q, p \wedge q, p \wedge \neg q, \alpha_{1}, \alpha_{2}, q \wedge \neg p, \alpha_{3}, \alpha_{4}\right\}$, where $\alpha_{1}=p \wedge \neg q \wedge \neg q$, $\alpha_{2}=p \wedge \neg q \wedge \neg q \wedge \neg q \alpha_{3}=q \wedge \neg p \wedge \neg p$ and $\alpha_{4}=q \wedge \neg p \wedge \neg p \wedge \neg p$. A profile for this pre-agenda is given in Table 7

We obtain $R_{M S A}(P)=\left\{\left\{q, \neg(p \wedge q), \neg(p \wedge \neg q), \neg \alpha_{1}, \neg \alpha_{2}, \neg(q \wedge \neg p), \neg \alpha_{3}, \neg \alpha_{4}\right\},\{p, \neg(p \wedge\right.$ $\left.q), \neg(p \wedge \neg q), \neg \alpha_{1}, \neg \alpha_{2}, \neg(q \wedge \neg p), \neg \alpha_{3}, \neg \alpha_{4}\right\},\left\{\neg(p \wedge q), \neg(p \wedge \neg q), \neg \alpha_{1}, \neg \alpha_{2}, \neg(q \wedge\right.$ $\left.\left.\neg p), \neg \alpha_{3}, \neg \alpha_{4}\right\}\right\}$. Consequently $T_{R_{M S A}}(P) \models p \vee q$.

To obtain $R_{M N A C}(P)$, we need to change the first three judgments of the first voter, obtaining the profile given in Table 7. This is the minimal change, since if either the second or the third agent change either their judgment on $p$ or their judgment on $q$, they have to change additional other three judgments. Hence we obtain

$R_{M N A C}(P)=\left\{\neg p, \neg q, \neg(p \wedge q), \neg(p \wedge \neg q), \neg \alpha_{1}, \neg \alpha_{2}, \neg(q \wedge \neg p), \neg \alpha_{3}, \neg \alpha_{4}\right\}$. We observe that $T_{R_{M N A C}}(P) \not \models p \vee q$.

To show that there exists a $P$ such that $T_{R_{M N A C}}(P) \nsubseteq T_{R_{M S A}}(P)$, consider the profile $P$ from Example 1. We have $T_{R_{M N A C}}(P) \models q$, see Example 7, but $T_{R_{M S A}}(P) \not \models q$, see Example 2.

Proposition $21 R_{M N A C}$ is incomparable with $R_{Y}, R_{R A}$, and $R_{M W A}$.

Proof: Consider the pre-agenda $[X]=\{p, q, p \wedge q\}$ and the following profile:

We obtain that $\neg(p \wedge q) \in T_{R_{M N A C}}(P)$, since $R_{M N A C}(P)=m\left(P^{\prime}\right) \cup m\left(P^{\prime \prime}\right)$, where $P^{\prime}$ and $P^{\prime \prime}$ are as in Tables 9 and 10.

On the other hand, we obtain: 


\begin{tabular}{c|ccccccccc} 
Voters & $p$ & $q$ & $p \wedge q$ & $p \wedge \neg q$ & $\alpha_{1}$ & $\alpha_{2}$ & $q \wedge \neg p$ & $\alpha_{3}$ & $\alpha_{4}$ \\
\hline $1 \times$ & - & - & - & - & - & - & - & - & - \\
$1 \times$ & + & - & - & + & + & + & - & - & - \\
$1 \times$ & - & + & - & - & - & - & + & + & + \\
\hline$m(P)$ & - & - & - & - & - & - & - & - & -
\end{tabular}

Table 8: After changing the first three judgments of the first agent.

\begin{tabular}{c|ccccccc} 
& & & & & & & \\
Voters & $p$ & $q$ & $p \wedge q$ & $r$ & $s$ & $r \wedge s$ & $t$ \\
\hline $1 \times$ & + & + & + & - & + & - & + \\
$3 \times$ & + & + & + & - & + & - & - \\
$4 \times$ & + & + & + & + & - & - & - \\
$2 \times$ & + & - & - & + & - & - & - \\
$4 \times$ & + & - & - & + & + & + & + \\
$4 \times$ & - & + & - & + & + & + & +
\end{tabular}

\begin{tabular}{r|ccc} 
Voters & $p$ & $q$ & $p \wedge q$ \\
\hline $1 \times$ & + & + & + \\
$1 \times$ & - & - & - \\
$1 \times$ & - & + & - \\
\hline$m(P)$ & - & + & -
\end{tabular}

Table 9: The profile $P^{\prime}$.

\begin{tabular}{r|ccc} 
Voters & $p$ & $q$ & $p \wedge q$ \\
\hline $1 \times$ & + & + & + \\
$1 \times$ & + & - & - \\
$1 \times$ & - & - & - \\
\hline$m(P)$ & + & - & -
\end{tabular}

Table 10: $P^{\prime \prime}$. 
- $R_{Y}=\{\{p\},\{q\},\{\neg(p \wedge q)\}\}$,

- $R_{R A}=\{\{p, \neg q, \neg(p \wedge q)\},\{p, \neg q, \neg(p \wedge q)\},\{p, q, p \wedge q\}\}$,

- $R_{M W A}=\{\{p, \neg q, \neg(p \wedge q)\},\{p, \neg q, \neg(p \wedge q)\},\{p, q, p \wedge q\}\}$.

Consequently $T_{R_{M N A C}}(P) \not \subset T_{R_{Z}}(P)$ for $Z \in\{Y, R A, M W A\}$.

To show that $T_{R_{Y}}(P) \not \subset T_{R_{M N A C}}(P)$ consider the profile $P$ from Example 1. As it can be observed from Example 7, $T_{R_{M N A C}}(P) \models p \wedge r$, but we can observe in Example 6 that for this profile $T_{R_{Y}}(P) \models \neg(p \wedge r)$. Furthermore, we can observe in Example 5 that $T_{R_{R A}}(P) \models \neg(p \wedge r)$.

To show that $T_{R_{M W A}}(P) \not \subset T_{R_{M N A C}}(P)$, consider again the pre-agenda of the proof of Proposition 20 and its corresponding profile $P$ given on Table 7. For this profile we get that $R_{M W A}(P)=\left\{p, q, p \wedge q, \neg(p \wedge \neg q), \neg \alpha_{1}, \neg \alpha_{2}, \neg(q \wedge \neg p), \neg \alpha_{3}, \neg \alpha_{4}\right\}$, since for this judgment set the weight is 17 , and for the remaining three other possible judgment sets the weights are: 14 for the set of the judgment sets of the second, and third agent and 16 for the judgment set $\left\{\neg p, \neg q, \neg(p \wedge q), \neg(p \wedge \neg q), \neg \alpha_{1}, \neg \alpha_{2}, \neg(q \wedge \neg p), \neg \alpha_{3}, \neg \alpha_{4}\right\}$. Consequently, $T_{R_{M W A}} \models p \vee q$. In the proof of Proposition 20 we show that $T_{R_{M N A C}}(P) \not \models$ $p \vee q$ for this profile.

Gathering all results, we get the diagram.

\section{Desirable properties for judgment aggregation rules}

We consider some important social choice-theoretic properties for judgment aggregation.

\subsection{Unanimity}

Unanimity is one of the most natural properties in social choice, guaranteeing that if all agents submit the same individual information to be aggregated then the aggregate is precisely that information. While unanimity is a relatively week property in voting theory and preference aggregation, satisfied by virtually all rules, in judgment aggregation, due to the logic relations between agenda issues, unanimity is not self assumed. The version of unanimity stating that if all agents submit the same judgment set then this judgment set is the aggregate is weak enough to be satisfied by all judgment aggregation rules we proposed. However, another version can be considered, the unanimity principle whether the unanimously selected judgment is included in the collective judgment sets. This is the version considered in the judgment aggregation literature. Dietrich and List [10] define the unanimity principle for judgment aggregation functions as follows.

\section{Definition 18 (unanimity principle [10])}

For all profiles $\left\langle A_{1}, \ldots, A_{n}\right\rangle$ in the domain of the aggregation function $f$ and all $\varphi \in X$, if $\varphi \in A_{i}$ for all individuals $i$, then $\varphi \in f\left(A_{1}, \ldots, A_{n}\right)$. 
Given that we consider nondeterministc rules, we have two versions of the unanimity principle, whether the unanimously approved formula must be in some collective judgment set (weak unanimity) or in all judgment sets (strong unanimity). Let $R$ be a judgment aggregation rule.

\section{Definition 19 (weak and strong unanimity)}

- $R$ satisfies weak unanimity if for every profile $P=\left\langle A_{1}, \ldots, A_{n}\right\rangle$ and all $\varphi \in X$, if $\varphi \in A_{i}$ for all $i$, then there exists a judgment set $A \in R(P)$ such that $\varphi \in A$.

- $R$ satisfies strong unanimity if for every profile $P=\left\langle A_{1}, \ldots, A_{n}\right\rangle$ and all $\varphi \in X$, if $\varphi \in A_{i}$ for all $i$, then $\varphi \in T_{R}(P)$.

It can be directly observed that the strong unanimity property implies weak unanimity property.

\subsection{Monotonicity}

In voting theory monotonicity is a standard property considered for voting rules. When a voting rule is monotonic, an improvement in the ranking of the winning alternative, ceteris paribus, does not diminish that alternative's likelihood of being a winner. When the purpose of aggregation is to select an alternative that is representative of the individual input, then it is desirable that additional support for an input should not make that input less likely to be the aggregate [28].

In judgment aggregation monotonicity has also been considered as a desirable property. There are three versions of monotonicity defined for judgment aggregation functions: monotonicity on an agenda issue as a property imposed on an aggregation function [24], monotonicity as a property imposed on a subset of the agenda (to address manipulability issues) [7], and monotonicity on a judgment set by [9]. The first property is the strongest, subsuming the other two.

We can define a monotonicity property for judgment aggregation rules following the property in [24].

Definition 20 (Monotonicity) Let $R$ be a judgment aggregation rule. Let $P=\left(A_{1}, \ldots, A_{i}, \ldots, A_{n}\right)$ be a profile for an agenda $X$ and $P^{\prime}=\left(A_{1}, \ldots, A_{i}^{\prime}, \ldots, A_{n}\right)$ its $i$-variant; $P \in \operatorname{Dom}(R)$ and $P^{\prime} \in \operatorname{Dom}(R)$. $R$ is monotonic when,

if there is a $\phi \in X$ such that

$$
\begin{aligned}
-\phi & \notin A_{i}, \\
-\phi & \in A_{i}^{\prime}, \text { and } \\
-\phi & \in T_{R}(P) \\
\text { then } \phi & \in T_{R}\left(P^{\prime}\right) \text { and } R\left(P^{\prime}\right)=R(P) .
\end{aligned}
$$

However, this monotonicity is a very strong property, since no constraints are put on $A_{i}^{\prime}$ with respect to $A_{i}$. Namely, it can happen that $A_{i}^{\prime} \cap A_{i}=\emptyset$ and for rules that do not satisfy independence, the collective judgment set can be affected on more issues 
than just $\phi$. To this end we can define a weaker monotonicity property and we consider whether out rules satisfy it. The intuition behind our new property, is closer to the intuition behind the monotonicity property as studied in voting theory. Namely, the ceteris paribus improvement in the support for a judgment that is already included in all collective judgment sets, should not diminish that judgments likelihood of being in all collective judgment sets. This is the property of insensitivity to reinforcement of collective judgements of collective judgements.

\section{Definition 21 (insensitivity to reinforcement)}

Let $P$ be a profile over $X$ and $\alpha \in X$. $P^{\prime}$ is called an $\alpha$-improvement of $P$ if $P^{\prime}=$ $\left(A_{i}^{\prime}, A_{-i}\right)$ where $(a) \neg \alpha \in A_{i}$ and $\left.A_{i}^{\prime}=\left(A_{i} \backslash\{\neg \alpha\}\right) \cup\{\alpha\}\right)$, and $(b) A_{i}^{\prime}$ is consistent. $R$ satisfies insensitivity to reinforcement of collective judgements iffor all profiles $P$ such that $\alpha \in T_{R}(P)$ and all $\alpha$-improvements $P^{\prime}$ of $P$, we have $R\left(P^{\prime}\right)=R(P)$.

It can be observed directly that if a rule $R$ is monotonic then it satisfies the insensitivity to reinforcement of collective judgments.

\subsection{Separability}

In voting theory, the separability property states that if an alternative is a winner, under a voting rule, for two distinct profiles under the same set of candidates, then that alternative is a winner, under the same voting rule, for the profile obtained by combining the two profiles. The property of separability is defined in [32], also defined as consistency in [33], and it is sometimes called reinforcement as well. This property is best known as one of the conditions, together with neutrality and anonymity, used by Young in his characterization of scoring social choice rules [33]. The voting rules that do not satisfy the separability property are subject to occurrences of the Simpson's paradox [1].

In judgment aggregation, the separability property is of interest as well. One reason is that the separability property is a natural requirement to make: if a judgment set is among the collective judgment sets for profile $P_{1}$ and for profile $P_{2}$, then it should be among the judgment sets for the combined profile $P$. Another reason can be found in the problem of implementation of judgment aggregation. Namely if a rule satisfies the separability property, then the aggregation process can be decentralized in some cases, separate groups of agents aggregating their profiles and then combining the output, which reduces the amount of information that needs to be exchanged between the agents.

\section{Definition 22 (separability)}

For all profiles $P_{1}, P_{2} \in \operatorname{Dom}(R)$, with $P_{1}=\left\langle A_{1}, \ldots, A_{n_{1}}\right\rangle$ and $P_{2}=\left\langle B_{1}, \ldots, B_{n_{2}}\right\rangle$, we define $P_{1}+P_{2}$ as the $n_{1}+n_{2}$-profile $\left\langle A_{1}, \ldots, A_{n_{1}}, B_{1}, \ldots, B_{n_{2}}\right\rangle$. Then we say that a rule $R$ satisfies separability if for every profiles $P_{1}, P_{2}$ such that $\alpha \in T_{R}\left(P_{1}\right)$ and $\alpha \in T_{R}\left(P_{2}\right)$, then $\alpha \in T_{R}\left(P_{1} \cup P_{2}\right)$.

\section{Results}

Proposition $22 R_{M S A}$ satisfies weak unanimity but not strong unanimity. 
Proof: Let $P$ be a profile on an agenda $X$, and $\varphi \in X$ such that all agents in $P$ agree on $\varphi$. There exists a maximal consistent agenda containing $\varphi$, and in this subagenda $P$ entails $\varphi$, therefore $R_{M S A}$ satisfies weak unanimity.

Consider the profile $P$ of the proof of Proposition 13 as a counter example. $R_{M S A}$ does not satisfy strong unanimity, because $a \notin T_{R_{M S A}}(P)$.

Proposition $23 R_{M C S A}$ does not satisfy weak (or strong) unanimity.

Proof: Consider again the profile $P$ of the proof of Proposition 13. The only maxcard consistent subagenda of $P$ contains $\neg a$ (and does not contain $a$ ). Consequently $R_{M C S A}$ does not even satisfy weak unanimity.

Proposition $24 R_{M W A}$ does not satisfy weak (or strong) unanimity.

Proof: See again the counterexample that can be found in [30], which we presented in the proof of Proposition 16.

Proposition $25 R_{R A}$ satisfies strong (and weak) unanimity.

Proof:Let $P$ be a profile and $Y_{P} \subseteq X$ be the subset of the agenda consisting of all elements on which there is unanimity among the agents. Because individual judgment sets are consistent, the conjunction of all elements of $Y$ is consistent. Now, when computing $R_{R A}(P)$, the elements of $Y$ are considered first, and whatever the order in which they are considered, they are included in $\delta$ because no inconsistency arises. Therefore, for all $\alpha \in Y_{P}$ and all $J \in R_{R A}(P)$, we have $\alpha \in J$.

Proposition $26 R_{Y}$ satisfies strong (and weak) unanimity.

Proof:Observe that if $\alpha$ is unanimously accepted by all agents in the set $N$, it is consequently unanimously selected by all consistent subsets of $N$.

Proposition $27 R^{d_{H}, \max }$ does not satisfy weak (or strong) unanimity.

Proof:Consider the pre-agenda

$[X]=\{a, b, c, d, \underbrace{(a \wedge b \wedge c \wedge d) \vee(\neg a \wedge \neg b \wedge \neg c \wedge \neg d)}_{\alpha}\}$,

and the profile $P=\left\langle A_{1}, A_{2}\right\rangle$ consisting of these two judgment sets: $A_{1}=\{a, b, c, d, \alpha\}$ and $A_{2}=\{\neg a, \neg b, \neg c, \neg d, \alpha\}$. The elements of $\Phi_{X}$ and their distances to $A_{1}$ and $A_{2}$ are given in Table 11. As it can be observed from the table, the $R^{d_{H}, \max }(P)$ selects all $A \in \Phi_{X}$ for which $\max \left(d_{H}\left(A, A_{1}\right), d_{H}\left(A, A_{2}\right)\right)=3$. For all such $A$ it holds that $\alpha \notin A$. Since $\alpha \in A_{1}$ and $\alpha \in A_{2}$, for this $P, R^{d_{H}, \max }$ does not satisfy the weak unanimity property.

Proposition $28 R_{M N A C}$ does not satisfy weak (nor strong) unanimity. 


\begin{tabular}{|r|c|c|c|}
\hline$A \in \Phi_{X}$ & $d_{H}\left(A, A_{1}\right)$ & $d_{H}\left(A, A_{2}\right)$ & $\max$ \\
\hline$\{a, b, c, d, \alpha\}$ & 0 & 4 & 4 \\
$\{a, b, c, \neg d, \neg \alpha\}$ & 2 & 4 & 4 \\
$\{a, b, \neg c, d, \neg \alpha\}$ & 2 & 4 & 4 \\
$\{a, b, \neg c, \neg d, \neg \alpha\}$ & 3 & 3 & 3 \\
$\{a, \neg b, c, d, \neg \alpha\}$ & 2 & 4 & 4 \\
$\{a, \neg b, c, \neg d, \neg \alpha\}$ & 3 & 3 & 3 \\
$\{a, \neg b, \neg c, d, \neg \alpha\}$ & 3 & 3 & 3 \\
$\{a, \neg b, \neg c, \neg d, \neg \alpha\}$ & 4 & 2 & 4 \\
$\{\neg a, b, c, d, \neg \alpha\}$ & 2 & 4 & 4 \\
$\{\neg a, b, c, \neg d, \neg \alpha\}$ & 3 & 3 & 3 \\
$\{\neg a, b, \neg c, d, \neg \alpha\}$ & 3 & 3 & 3 \\
$\{\neg a, b, \neg c, \neg d, \neg \alpha\}$ & 4 & 2 & 4 \\
$\{\neg a, \neg b, c, d, \neg \alpha\}$ & 3 & 3 & 3 \\
$\{\neg a, \neg b, c, \neg d, \neg \alpha\}$ & 4 & 2 & 4 \\
$\{\neg a, \neg b, \neg c, d, \neg \alpha\}$ & 4 & 2 & 4 \\
$\{\neg a, \neg b, \neg c, \neg d, \alpha\}$ & 4 & 0 & 4 \\
\hline
\end{tabular}

Table 11: The max of Hamming distances from an element in the set $\Phi_{X}$ to each of the agent's judgment sets.

Proof: Again consider the agenda and profile $P$ in the proof of Proposition 16. Since $R_{M N A C}(P)=R_{M W A}(P)$, this example of a profile is a counter example for $R_{M N A C}$ satisfying weak unanimity as well.

Proposition $29 R_{M S A}, R_{M C S A}, R_{M W A}$ and $R_{R A}$ are insensitive to reinforcement of collective judgements.

\section{Proof:}

1. We consider $R_{M S A}$. Assume that $\alpha \in T_{R_{M S A}}(P)$. Let $X^{\prime} \subseteq X$ be a maximal agenda for which $P^{\downarrow X^{\prime}}$ is majority-consistent. Because $\alpha \in T_{R_{M S A}}(P)$, we must have $\alpha \in X^{\prime}$. Obviously, $P^{\prime \backslash X^{\prime}}$ is majority-consistent as well and moreover $m\left(P^{\prime \backslash X^{\prime}}\right)=$ $m\left(P^{\downarrow X^{\prime}}\right)(1)$. Moreover, it entails that all maximal majority-consistent subagenda for $P^{\prime}$ contain some maximal majority-consistent subagenda for $P^{\prime}(2)$. Now, let $X^{\prime} \subseteq X$ be a maximal agenda for which $P^{\prime \downarrow} X^{\prime}$ is majority-consistent. If $\alpha \notin$ $m\left(P^{\prime \backslash X^{\prime}}\right)$ then a fortiori $\alpha \notin m\left(P^{\downarrow X^{\prime}}\right)$, which contradicts (2). Therefore, $\alpha \in$ $m\left(P^{\prime} \backslash X^{\prime}\right)$, and because of (2), it is also a maximal majority-consistent subagenda for $P$. We have shown that the maximal majority-consistent subagendas for $P$ and $P^{\prime}$ coincide, therefore $R_{M S A}(P)=R_{M C S A}\left(P^{\prime}\right)$. The proof for $R_{M C S A}$ is similar.

2. Now, we consider $R_{R A}$. Let $\alpha \in X$ and assume that $\alpha \in T_{R_{R A}}(P)$. Then all subagendas in $R_{R A}(P)$ contains $\alpha$. Let $P^{\prime}$ be an $\alpha$-improvement of $P$. Then $N\left(P^{\prime}, \alpha\right)>N(P, \alpha), N\left(P^{\prime}, \neg \alpha\right)<N(P, \neg \alpha)$, whereas for all $\varphi \neq \alpha, \neg \alpha, N\left(P^{\prime}, \varphi\right)=$ $N(P, \varphi)$. Note that in $\geq_{P^{\prime}}, \alpha$ appears either at an earlier position or in the same 
position as in $\geq_{P}$. Therefore, if $\succ^{\prime}$ be an order refining $\geq_{P^{\prime}}$, when $\alpha$ is considered in $\succ$, it must be consistent with $D$, otherwise there would be an order $\succ$ refining $\geq_{P}$ resulting in a subagenda not containing $\alpha$. Therefore $\alpha$ belongs to all subagendas in $R A\left(P^{\prime}\right)$.

3. We consider $R_{M W A}$ i.e., $R^{d_{H}, \Sigma}$. Let $P$ be a profile $P=\left(A_{1}, \ldots, A_{k}, \ldots, A_{n}\right)$ and its a $\alpha$-reinforcement, a profile $P^{\prime}=\left(A_{1}^{\prime}, \ldots, A_{k}^{\prime}, \ldots, A_{n}^{\prime}\right)=\left(A_{1}, \ldots, A_{k}^{*}, \ldots, A_{n}\right)$. Let $\Phi_{X}$ be the set of all consistent and complete judgment sets over an agenda $X$. Let us define $D(A, P)=\sum_{i=1}^{n} d_{H}\left(A, A_{i}\right)$.

We have the following assumptions:

- $\alpha \notin A_{k}$,

- $\alpha \in A_{k}^{*}$,

- for all $\psi \in X, \psi \notin\{\alpha, \neg \alpha\}$ it holds $\psi \in A_{k}$ iff $\psi \in A_{k}^{*}$,

- $\alpha \in A$, foralla $\in R^{d_{H}, \Sigma}(P)$.

We first show that all the judgment sets $A \in R^{d_{h}, \Sigma}(P)$ are such that $A \in R^{d_{h}, \Sigma}\left(P^{\prime}\right)$ and that there exists no $A^{\prime} \in \Phi_{X}$ such that: $\alpha \in A^{\prime}, A^{\prime} \notin R^{d_{h}, \Sigma}(P)$, but $A^{\prime} \in$ $R^{d_{h}, \Sigma}\left(P^{\prime}\right)$.

Let the score of the winner judgment sets $A$ for $P$ be c, namely let $c=\sum_{i=1}^{n} d_{H}\left(A, A_{i}\right)$, for all $A \in R^{d_{h}, \Sigma}(P)$. We have that, for all $A^{\prime} \in \Phi_{X}$, when the cardinality of the pre-agenda is $m$ :

$d_{H}\left(A^{\prime}, A_{k}\right)=m-\left|A_{k} \cap A^{\prime}\right|$, $d_{H}\left(A^{\prime}, A_{k}^{*}\right)=m-\left|A_{k}^{*} \cap A^{\prime}\right|$.

Let $A^{\prime} \in \Phi_{X}$ be such that $\alpha \in A^{\prime}$. Since $d_{H}\left(A_{k}, A_{k}^{*}\right)=1$, we have that $\left|A_{k} \cap A^{\prime}\right|-$ $\left|A_{k}^{*} \cap A^{\prime}\right|=1$. Hence $d_{H}\left(A^{\prime}, A_{k}\right)=1+d_{H}\left(A^{\prime}, A_{k}^{*}\right.$ and

$$
D\left(A^{\prime}, P\right)=1+D\left(A^{\prime}, P^{\prime}\right) .
$$

For all the winners $A$ for $P$, we obtain that $D(A, P)=1+D\left(A, P^{\prime}\right)$, hence

$$
D\left(A, P^{\prime}\right)=c-1 \text {. }
$$

If an $A^{\prime} \notin R^{d_{h}, \Sigma}(P)$, then $D\left(A^{\prime}, P\right)>c$ and due to $1, D\left(A^{\prime}, P^{\prime}\right)>c-1$. We can conclude that there is no $A^{\prime} \in \Phi_{X}$ such that $\alpha \in A^{\prime}$ and $A^{\prime} \notin R^{d_{h}, \Sigma}(P)$ but $A^{\prime} \in$ $R^{d_{h}, \Sigma}\left(P^{\prime}\right)$.

We now show that there exists no $A^{\prime \prime} \in \Phi_{X}$ such that $\alpha \notin A^{\prime \prime}$ and $A^{\prime \prime} \notin R^{d_{h}, \Sigma}(P)$ but $A^{\prime \prime} \in R^{d_{h}, \Sigma}\left(P^{\prime}\right)$. We construct a proof by contradiction, starting with the assumption that there exists such a $A^{\prime \prime} \in R^{d_{h}, \Sigma}\left(P^{\prime}\right)$.

Since $A^{\prime \prime} \notin R^{d_{h}, \Sigma}(P)$, we obtain

$$
D\left(A^{\prime \prime}, P\right)>c \text {. }
$$




\begin{tabular}{c|ccccc|cccc} 
Voters & $p$ & $q$ & $p \wedge q$ & $r$ Voters & $p$ & $q$ & $p \wedge q$ & $r$ \\
\hline $2 \times$ & + & + & + & + & $2 \times$ & + & + & + & + \\
$2 \times$ & + & - & - & + & $2 \times$ & + & - & - & + \\
$1 \times$ & + & - & - & + & $1 \times$ & - & - & - & + \\
$4 \times$ & - & + & - & - & $4 \times$ & - & + & - & - \\
\hline$m(P)$ & + & + & - & + & $m(P)$ & - & + & - & +
\end{tabular}

Table 12: $P$ on the left, and the $\neg p$-reinforcement $P^{\prime}$ on the right.

Since $\alpha \in A_{k}^{*}$ and $\alpha \notin A^{\prime}$, we obtain $d_{H}\left(A^{\prime \prime}, A_{k}\right)<d_{H}\left(A^{\prime \prime}, A_{k}^{*}\right)$ and consequently

$$
D\left(A^{\prime \prime}, P\right)<D\left(A^{\prime \prime}, P^{\prime}\right) \text {. }
$$

Putting together inequalities 3 and 4 we obtain

$$
D\left(A^{\prime \prime}, P^{\prime}\right)>c
$$

However, the inequality 5 and inequality 2 are contradictory with the assumption that $A^{\prime \prime} \notin R^{d_{h}, \Sigma}(P)$. This completes the proof that $R^{d_{h}, \Sigma}$ is insensitive to reinforcement of collective judgements.

Proposition $30 R_{Y}$ is not insensitive to reinforcement of collective judgements.

Proof: We show that although it holds that it is not the case that $R_{Y}(P) \neq R_{Y}\left(P^{\prime}\right)$ when $\alpha \in T_{Y}(P)$ and $P^{\prime}$ is a $\alpha$-reinforcement of $P$. We use a proof by counter example. Let the pre-agenda be $[X]=\{p, q, p \wedge q, r\}$. Consider the profile $P$ of 9 voters:

Consider the profile $P$ in Table 12. $P$ is not majority-consistent, but removing any voter who has $p$ in her judgment set suffices to restore consistency, therefore $R_{Y}(P)=$ $\{q, \neg(p \wedge q)\}$. Consider the $\neg p$-reinforcement profile $P^{\prime}$, Table 12 right-most. Now $R_{Y}\left(P^{\prime}\right)=\{\neg p, q, \neg(p \wedge q), r\}$. Observe that although $\neg p \in T_{Y}(P)$ and $\neg p \in T_{Y}\left(P^{\prime}\right)$, $R_{Y}(P) \neq_{T} R_{Y}\left(P^{\prime}\right)$.

Proposition $31 R^{d_{H}, \max }$ is not insensitive to reinforcement of collective judgements.

Proof: Consider the pre-agenda $[X]=\{p \wedge r, p \wedge q, q, t\}$, the profile $P$ for three agents:

\begin{tabular}{c|cccc} 
Voters & $p \wedge r$ & $p \wedge q$ & $q$ & $t$ \\
\hline $1 \times$ & - & + & - & + \\
$1 \times$ & + & - & - & - \\
$1 \times$ & - & - & + & +
\end{tabular}

and its $\neg(p \wedge q)$-reinforcement (in the first voter's judgment set) $P^{\prime}$ :

\begin{tabular}{c|cccc} 
voters & $p \wedge r$ & $p \wedge q$ & $q$ & $t$ \\
\hline 1 & - & - & - & + \\
2 & + & - & - & - \\
3 & - & - & + & +
\end{tabular}


As it can be observed from Table 13, $\neg(p \wedge q) \in T_{R^{d_{H}, \max }}(P)$, since $R^{d_{H}, \max }(P)=\{\{\neg(p \wedge r), \neg(p \wedge q), \neg q, \neg t\},\{\neg(p \wedge r), \neg(p \wedge q), \neg q, t\},\{p \wedge r, \neg(p \wedge$ $q), \neg q, t\}\}$. However, as it can be observed from Table 14, $R^{d_{H}, \max }\left(P^{\prime}\right)=\{\{\neg(p \wedge$ $r), \neg(p \wedge q), \neg q, \neg t\},\{\neg(p \wedge r), \neg(p \wedge q), \neg q, t\},\{p \wedge r, \neg(p \wedge q), \neg q, t\},\{\neg(p \wedge r), \neg(p \wedge$ $q), q, \neg t\}\}$. Thus, $\neg(p \wedge q) \in T_{R^{d_{H}, \max }}\left(P^{\prime}\right)$, but $R^{d_{H}, \max }(P) \neq R^{d_{H}, \max }\left(P^{\prime}\right)$. Furthermore, since $\neg q \in T_{R^{d_{H}, \max }}(P)$, but $\neg q \notin T_{R^{d_{H}, \max }}\left(P^{\prime}\right)$, we obtain that $R^{d_{H}, \max }(P) \neq_{T}$ $R^{d_{H}, \max }\left(P^{\prime}\right)$.

\begin{tabular}{|cr|c|c|c|c|}
\hline$A \in \Phi_{X}$ & & $d_{H}\left(A, A_{5}\right)$ & $d_{H}\left(A, A_{8}\right)$ & $d_{H}\left(A, A_{4}\right)$ & $\max$ \\
\hline$A_{1}$ & $\{\neg(p \wedge r), \neg(p \wedge q), \neg q, \neg t\}$ & 2 & 1 & 2 & 2 \\
\hline$A_{2}$ & $\{\neg(p \wedge r), \neg(p \wedge q), \neg q, t\}$ & 1 & 2 & 1 & 2 \\
\hline$A_{3}$ & $\{\neg(p \wedge r), \neg(p \wedge q), q, \neg t\}$ & 3 & 2 & 1 & 3 \\
\hline$A_{4}$ & $\{\neg(p \wedge r), \neg(p \wedge q), q, t\}$ & 2 & 3 & 0 & 3 \\
\hline$A_{5}$ & $\{\neg(p \wedge r), p \wedge q, \neg q, t\}$ & 0 & 3 & 2 & 3 \\
\hline$A_{6}$ & $\{\neg(p \wedge r), p \wedge q, q, \neg t\}$ & 2 & 3 & 2 & 3 \\
\hline$A_{7}$ & $\{\neg(p \wedge r), p \wedge q, q, t\}$ & 1 & 4 & 1 & 4 \\
\hline$A_{8}$ & $\{p \wedge r, \neg(p \wedge q), \neg q, \neg t\}$ & 3 & 0 & 3 & 3 \\
\hline$A_{9}$ & $\{p \wedge r, \neg(p \wedge q), \neg q, t\}$ & 2 & 1 & 2 & 2 \\
\hline$A_{10}$ & $\{p \wedge r, p \wedge q, q, \neg t\}$ & 3 & 2 & 3 & 3 \\
\hline$A_{11}$ & $\{p \wedge r, p \wedge q, q, t\}$ & 2 & 3 & 2 & 3 \\
\hline
\end{tabular}

Table 13: The max of Hamming distances from an element in the set $\Phi_{X}$ to each of the agent's judgment sets in profile $P$.

\begin{tabular}{|cr|c|c|c|c|}
\hline$A \in \Phi_{X}$ & & $d_{H}\left(A, A_{2}\right)$ & $d_{H}\left(A, A_{8}\right)$ & $d_{H}\left(A, A_{4}\right)$ & $\max$ \\
\hline$A_{1}$ & $\{\neg(p \wedge r), \neg(p \wedge q), \neg q, \neg t\}$ & 2 & 1 & 2 & 2 \\
\hline$A_{2}$ & $\{\neg(p \wedge r), \neg(p \wedge q), \neg q, t\}$ & 1 & 2 & 1 & 2 \\
\hline$A_{3}$ & $\{\neg(p \wedge r), \neg(p \wedge q), q, \neg t\}$ & 1 & 2 & 1 & 2 \\
\hline$A_{4}$ & $\{\neg(p \wedge r), \neg(p \wedge q), q, t\}$ & 0 & 3 & 0 & 3 \\
\hline$A_{5}$ & $\{\neg(p \wedge r), p \wedge q, \neg q, t\}$ & 2 & 3 & 2 & 3 \\
\hline$A_{6}$ & $\{\neg(p \wedge r), p \wedge q, q, \neg t\}$ & 1 & 3 & 2 & 3 \\
\hline$A_{7}$ & $\{\neg(p \wedge r), p \wedge q, q, t\}$ & 1 & 4 & 1 & 4 \\
\hline$A_{8}$ & $\{p \wedge r, \neg(p \wedge q), \neg q, \neg t\}$ & 3 & 0 & 3 & 3 \\
\hline$A_{9}$ & $\{p \wedge r, \neg(p \wedge q), \neg q, t\}$ & 2 & 1 & 2 & 2 \\
\hline$A_{10}$ & $\{p \wedge r, p \wedge q, q, \neg t\}$ & 2 & 2 & 3 & 3 \\
\hline$A_{11}$ & $\{p \wedge r, p \wedge q, q, t\}$ & 1 & 3 & 2 & 3 \\
\hline
\end{tabular}

Table 14: The max of Hamming distances from an element in the set $\Phi_{X}$ to each of the agent's judgment sets in profile $P^{\prime}$.

For the remaining rule, namely $R_{M N A C}$, we have no result. We conjecture that it is insensitive to reinforcement of collective judgements.

Lastly, we consider separability. We establish a general result which shows that 
majority-preservation and rule separability are incompatible. This result can be seen as the judgment aggregation counterpart as the result stating that any Condorcet-consistent voting rule violates reinforcement, see Theorem 9.2 [pg.237, [27]].

Proposition 32 Any majority-preserving judgment aggregation rule violates separability.

Proof: Let $R$ be a majority-preserving rule, and assume furthermore that $R$ satisfies separability. Let $[X]=\{p, q, p \vee q\}$, and $P$ the 10 -voter profile as follows:

\begin{tabular}{c|ccc} 
voters & $p$ & $q$ & $p \vee q$ \\
\hline 1,2 & + & + & + \\
3,4 & - & + & + \\
5,6 & + & - & + \\
$7,8,9,10$ & - & - & -
\end{tabular}

Consider the two subprofiles $P_{1}$ consisting of voters $\{1,3,4,7,8\}$ and $P_{2}$ consisting of voters $\{2,5,6,9,10\} . P_{1}$ and $P_{2}$ are majority-consistent, with $m\left(P_{1}\right)=\{\neg p, q, p \vee$ $q\}$ and $m\left(P_{2}\right)=\{p, \neg q, p \vee q\}$. Since $R$ is majority-preserving, we have $R\left(P_{1}\right)=$ $\{\{p, \neg q, p \vee q\}\}$ and $R\left(P_{2}\right)=\{\{q, \neg p, p \vee q\}\}$; therefore, $p \leftrightarrow \neg q \in T_{R}\left(P_{1}\right)$ and $p \leftrightarrow$ $\neg q \in T_{R}\left(P_{2}\right)$, from which, by separability, (1) $p \leftrightarrow \neg q \in T_{R}\left(P_{1} \cup P_{2}\right)=T_{R}(P)$.

Consider now the two subprofiles $P_{3}$ consisting of voters $\{1,2,3\}$ and $P_{4}$ consisting of voters 4 to 10. $P_{3}$ and $P_{4}$ are majority-consistent, with $m\left(P_{3}\right)=\{p, q, p \wedge q\}$ and $m\left(P_{4}\right)=\{\neg p, \neg q, \neg p \wedge \neg q\}$. Since $R$ is majority-preserving, we have $R\left(P_{3}\right)=$ $\{\{p, q, p \wedge q\}\}$ and $R\left(P_{4}\right)=\{\{\neg p, \neg q, \neg p \wedge \neg q\}\}$; therefore, $p \leftrightarrow q \in T_{R}\left(P_{3}\right)$ and $p \leftrightarrow \neg q \in T_{R}\left(P_{4}\right)$, from which, by separability, (3) $p \leftrightarrow q \in T_{R}\left(P_{1} \cup P_{2}\right)=T_{R}(P)$, in contradiction with (1).

As a corollary, all our rules except $R^{d_{H}, \max }$ violate separability.

Corollary 1 None of the aggregation rules $R_{Y}, R_{M S A}, R_{M C S A}, R_{M W A}, R_{R A}$ and $R_{M N A C}$ satisfies separability.

The only one of our rules which is not majority-preserving is $R^{d_{H} \text {, max }}$. However, this one does not satisfy separability either, which shows that it seems extremely difficult to find a reasonable judgment aggregation rule that satisfies separability.

Proposition $33 R^{d_{H}, \max }$ does not satisfy separability.

Proof: Let $[X]=\{p, q, r, p \rightarrow(q \wedge r)\}$, and the 5-voter profile $P$ :

\begin{tabular}{c|cccc} 
voters & $p$ & $q$ & $r$ & $p \rightarrow(q \wedge r)$ \\
\hline $1,2,3$ & + & + & - & - \\
4,5 & + & + & + & +
\end{tabular}

Consider also the two sub-profiles $P_{1}$ consisting of voters 1,2 and 3 , and $P_{2}$ consisting of voters 4 and 5. Observe that $R^{d_{H}, \max }\left(P_{1}\right)=\{\{p, q, \neg r, \neg(p \rightarrow(q \wedge r))\}\}$ and $R^{d_{H}, \max }\left(P_{2}\right)=\{\{p, q, r, p \rightarrow(q \wedge r)\}\}$, thus $p \in T_{R^{d_{H}, \max }}\left(P_{1}\right)$ and $p \in T_{R^{d_{H}, \max }}\left(P_{2}\right)$. However, $R^{d_{H}, \max }(P)=\{\{p, q, \neg r, \neg(p \rightarrow(q \wedge r))\},\{p, q, r, p \rightarrow(q \wedge r)\},\{\neg p, q, \neg r, p \rightarrow$ $(q \wedge r)\},\{p, \neg q, r, \neg(p \rightarrow(q \wedge r))\}\}$, therefore $p \notin T_{R^{d_{H}, \max }}(P)$. 


\section{Conclusion}

We have studied seven judgment aggregation rules. Four of them had already been defined elsewhere - three of them in [25] — but had not been studied from the point of views of the properties we considered here. The other three $\left(R_{Y}, R_{M S A}\right.$ and $\left.R_{R A}\right)$ are totally new. We have extensively studied the inclusion (or noninclusion) relationships between these 7 rules (Table 7). Then we have focuses on three desirable properties of judgment aggregation rules and identified which of our rules satisfy them (with one missing result). The table below summarizes the results.

\begin{tabular}{|c|l|l|l|l|c|}
\hline & $\begin{array}{l}\text { Majority } \\
\text { Preservation }\end{array}$ & $\begin{array}{l}\text { Weak } \\
\text { Unananimity }\end{array}$ & $\begin{array}{l}\text { Strong } \\
\text { Unaninimity }\end{array}$ & Reinforcement & Separability \\
\hline$R_{Y}$ & $\checkmark$ & $\checkmark$ & $\checkmark$ & no & no \\
\hline$R_{M S A}$ & $\checkmark$ & $\checkmark$ & no & $\checkmark$ & no \\
\hline$R_{M C S A}$ & $\checkmark$ & no & no & $\checkmark$ & no \\
\hline$R_{M W A}$ & $\checkmark$ & no & no & $\checkmark$ & no \\
\hline$R_{R A}$ & $\checkmark$ & $\checkmark$ & $\checkmark$ & $\checkmark$ & no \\
\hline$R^{d_{H}, \max }$ & no & no & no & no & no \\
\hline$R_{M N A C}$ & $\checkmark$ & no & no & $?$ & no \\
\hline
\end{tabular}

Table 15: Summary of the results for the social theoretic properties of the judgment aggregation rules.

Obviously, many other meaningful properties should be investigated, and even if it would be premature to make a conclusion given the few properties we considered, we may still observe that $R_{R A}$ Pareto-dominates all other rules in terms if the properties satisfied.

It is also vital to consider the computational properties of the rules we introduce, such as complexity of winner determination (see [14]). Another issue that we plan to investigate in the future is the strategic aspect of our aggregation rules (as done in judgment aggregation [7]), that is, how voters can strategically submit insincere judgment sets in order to induce specific outcomes. These computational and manipulation issues will be addressed in a future paper.

\section{Acknowledgements}

We thank Nicolas Maudet as well as the anonymous reviewers for helpful comments. Jérôme Lang thanks the ANR project ComSoc (ANR-09-BLAN-0305).

\section{References}

[1] C. R. Blyth. On Simpson's Paradox and the Sure-Thing Principle. Journal of the American Statistical Association, 67(338):364-366, 1972. 
[2] S. Brams and P. Fishburn. Voting procedures. In K. Arrow, A. Sen, and K. Suzumura, editors, Handbook of Social Choice and Welfare, chapter 4. Elsevier, 2004.

[3] S. Brams, D. M. Kilgour and R. Sanver. A minimax procedure for electing committees. Public Choice, 132(3-4): 401-420, 2007.

[4] B. Chapman. Rational aggregation. Politics, Philosophy \& Economics, 1(3): 337-354, 2002.

[5] F. Dietrich. Judgment aggregation: (im)possibility theorems. Economic Theory, 126:286-298, 2006.

[6] F. Dietrich. The possibility of judgment aggregation on agendas with subjunctive implications. Journal of Economic Theory, 145(2):603 - 638, 2010.

[7] F. Dietrich and C. List. Strategy-proof judgment aggregation. Economics and Philosophy, 23: 269-300, 2007.

[8] F. Dietrich and C. List. Judgment aggregation by quota rules: Majority voting generalized. Journal of Theoretical Politics, 4(19):391 - 424, 2007.

[9] F. Dietrich and C. List. Judgment aggregation without full rationality. Social Choice and Welfare, 31(1):15-39, June 2008.

[10] F. Dietrich and C. List. A liberal paradox for judgment aggregaton. Social Choice and Welfare, 31(1):59-78, June 2008.

[11] F. Dietrich and P. Mongin. The premisse-based approach to judgment aggregation. Journal of Economic Theory, 145(2):562 - 582, 2010.

[12] E. Dokow and R. Holzman Aggregation of binary evaluations with abstentions. Journal of Economic Theory, 145(2):544-561, 2010.

[13] E. Elkind, P. Faliszewski and A. Slinko On distance rationalizability of some voting rules. Proceedings of the 12th Conference on Theoretical Aspects of Rationality and Knowledge (TARK '09), 108-117, 2009.

[14] U. Endriss, U. Grandi, and D. Porello. Complexity of winner determination and strategic manipulation injudgment aggregation. In COMSOC-10, 2009.

[15] P. Gärdenfors. A representation theorem for voting with logical consequences. Economics and Philosophy", 22:181-190, 2006.

[16] S. Konieczny, J. Lang, and P. Marquis. Da2 merging operators. Artificial Intelligence Journal, 157:45-79, 2004.

[17] S. Konieczny and R. Pino-Pérez. Merging with integrity constraints. Lecture Notes in Computer Science, 1638/1999:233-244, January 1999.

[18] S. Konieczny and R. Pino-Pérez. Logic Based Merging Journal of Philosophical Logic, 40(2), 239-270, 2011. 
[19] L.A. Kornhauser and L.G. Sager. The one and the many: Adjudication in collegial courts. California Law Review, 81:1-51, 1993.

[20] J. Lang, G. Pigozzi, M. Slavkovik and L. van der Torre. Judgment aggregation rules based on minimization. Proceedings of the 13th Conference on Theoretical Aspects of Rationality and Knowledge (TARK-2011), Groningen, The Netherlands, July 12-14, 2011, ACM, 238-246.

[21] N. Li. Decision paths in sequential non-binary judgment aggregation. Technical report, Universitat Autònoma de Barcelona, 2010.

[22] C. List. A model of path-dependence in decisions over multiple propositions. American Political Science Review, 3(98):495 - 513, 2004.

[23] C. List and Philip Pettit. Aggregating sets of judgments: Two impossibility results compared. Synthese, 140(1/2):pp. 207-235, 2004.

[24] C. List and C. Puppe. Judgment aggregation: A survey. In P. Anand, C. Puppe, and P. Pattanaik, editors, Oxford Handbook of Rational and Social Choice. Oxford, 2009.

[25] M. Miller and D. Osherson. Methods for distance-based judgment aggregation. Social Choice and Welfare, 32(4):575 - 601, 2009.

[26] P. Mongin. Factoring out the impossibility of logical aggregation. Journal of Economic Theory, 141:100 - 113, 2004.

[27] H. Moulin. Axioms of cooperative decision making, Econometric Society Monographs 15, Cambridge University Press, 1991.

[28] H. Nurmi. Monotonicity and its cognates in the theory of choice. Public Choice, 121(1/2):25-49, 2004.

[29] G. Pigozzi. Belief merging and the discursive dilemma: an argument-based account to paradoxes of judgment aggregation. Synthese, 152(2):285-298, 2006.

[30] G. Pigozzi, M. Slavkovik, and L. van der Torre. A complete conclusion-based procedure for judgment aggregation. In First International Conference on Algorithmic Decision Theory Proceedings, pages 1-13, 2009.

[31] M. Schulze. A new monotonic and clone-independent single-winner election method. Voting Matters, 17:9-19, 2003.

[32] J.H. Smith. Aggregation of preferences with variable electorate. Econometrica, 41(6):1027-1041, 1973.

[33] H. P. Young. Social choice scoring functions. SIAM Journal on Applied Mathematics, 28(4):824-838, 1975. 